\title{
Environment reconstruction and navigation with electric sense based on kalman filter
}

\author{
Vincent Lebastard, Christine Chevallereau’, Alexis Girin* \\ Noël Servagent; Pol-Bernard Gossiaux ${ }^{\ddagger}$ and Frédéric Boyer*
}

November 13, 2012

\begin{abstract}
Electric fish sense the perturbations of a self generated electric field through their electroreceptive skin. This sense allows them to navigate and reconstruct their environment in conditions where vision and sonar cannot work. In this article, we use a sensor inspired by this sense to address both problems of locating and estimating the size of small objects (electrolocation) and navigating in a tank. . Based on a Kalman filter, any small object in the surroundings of the motion controlled sensor can be modeled as an equivalent sphere whose location is well estimated by the filter. As a first application to the problem of navigation, the filter is included into a closed feedback loop in order to achieve wall following in a tank. Our experimental results demonstrate the feasibility of this approach.
\end{abstract}

\section{Introduction}

Developed by several hundreds of fish species who have co-evolved on both African and SouthAmerican continents, the electric sense was discovered by Lissman in 1958 [LM58]. In African fish Gnathonemus Petersii for instance, the fish first polarizes its body with an electric organ discharge (EOD) located at the base of its tail (Figure 1). This polarization is applied in short pulses, generating a dipolar shaped electric field around the fish which is then distorted by the objects in its surroundings. The fish "measures" the distortion of the electric fields using an array of electro-receptors distributed along its body and infers an image of its surroundings through neural processing intensively studied by neurobiologists [CBGB98, vdE06, $\mathrm{EBM}^{+} 08, \mathrm{EvdE11}$ ]. In order to study the environment modalities sensed by the fish, biologists have developed many neuroethological experiments in which fish are trained to recognize a given shape [vdESG ${ }^{+}$98, vdE99].

Electric fish can easily navigate in the dark or/and in turbid waters of confined unstructured environments such as the roots of the trees in the flooded tropical forests which are their natural habitat. Neither vision or technologies such as sonar can work in these conditions but the electric sense is well-adapted for this niche. Based on this fact, McIver et al have recently exploited an experimental made of four point electrodes placed at the apices of a rhombus in a rigid moving frame

${ }^{*}$ F. Boyer, A. Girin and V. Lebastard are with IRCCyN-Ecole des Mines de Nantes, 4, rue Alfred Kastler B.P. 20722 - 44307 Nantes Cedex 3 France.

${ }^{\dagger}$ C. Chevallereau is with IRCCyN-CNRS, Ecole Centrale de Nantes, 1 rue de la Noë, 44000 Nantes, France.

${ }^{\ddagger}$ P.B. Gossiaux, Noël Servagent are with SUBATECH-Ecole des Mines de Nantes, 4, rue Alfred Kastler B.P. 20722 - 44307 Nantes Cedex 3. 
driven by a Cartesian robot [SLM07]. In this device, two electrodes located at opposite apices of the rhombus are polarized in voltage and play the role of the electric organ of discharge of the fish while the other two electrodes play the role of receivers between which the voltage is measured. Using Cartesian motion control of this sensor in a tank, they successfully located small spheres through off-line particle filtering [SLM07, SLM08]. However, the body geometry of this sensor ideally reduces to four point electrode, limiting its implementation on a real autonomous underwater vehicle. Using an alternative sensor technology based on the measurement of current, Kalman filtering technics have been applied to the reconstruction of the surroundings of an underwater vehicle of realistic geometry [BBG08, $\left.\mathrm{LCA}^{+} 10\right]$. In [BBG08] the surroundings are constituted of insulating infinite walls while in $\left[\mathrm{LCA}^{+} 10\right]$, the case of small (insulating and conducting) objects is tackled. However, all these results have hither to been restricted to simulations. This article presents experiments with these techniques in the context of localization and navigation these techniques for the implementation of the electric sense. In controlled experimental conditions, the problem of reconstruction of the state of small objects and tank navigation is addressed. We define "reconstruction", as the estimation of the size and location of these objects in the sensor frame. Because the electrical equations cannot be integrated analytically for an object of arbitrary shape [SYOPA04], we confine our investigations to the case of spherical objects. However, we will see that objects of other shape such as cubes, can be modeled by equivalent spheres (and "encapsulated" in them). As far as the problem of navigation is concerned, we propose a model based approach in which the filter is included in a global feedback loop. We will illustrate the case where the assigned task consists of following the walls of a tank.

Finally, until now, electric sense has never been applied to (at least to our knowledge): 1) the problem of on-line reconstruction of scenes comprising walls, corners and small objects, 2) the application of Kalman filtering technics to this problem and 3) the technological principle of our electric sensor based on the current measurements. Furthermore, the application of Kalman filtering technics to this problem as well as the technological principle of our electric sensor (based on the current measurements) also contribute to the novelty of the approach. Lastly, in spite of the long way that separates the results presented here in simplified conditions (few objects in a tank) from the realization of the electric sense on a real autonomous vehicle, we believe that our results have the potential to guide future research making use of this unique underwater sensing modality.
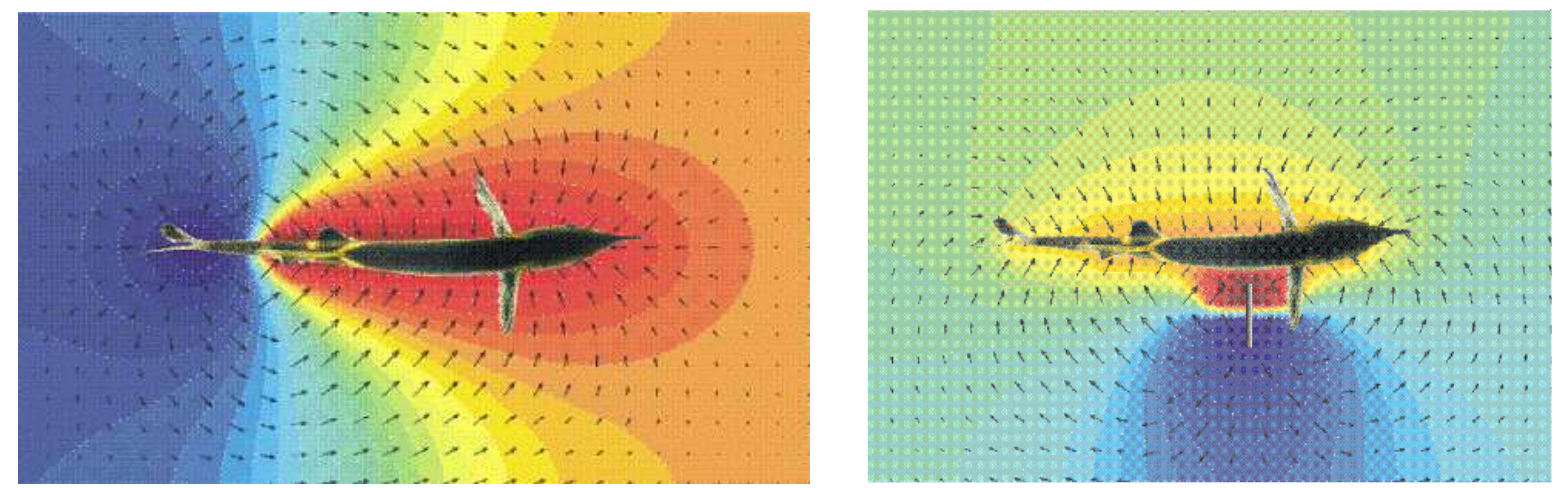

Figure 1: The electric fish, here the Gnathonemus Petersii. (left) The basal electric field, (right) the electric field is distorted by the presence of an object on its right.

The article is structured as follows. First, we briefly present the sensor technology (section 2) 
and experimental conditions. We then address models for perception and locomotion in section 3 . A solution to the problem of reconstructing a model of the surroundings is detailed in section 4 . Localization and reconstruction are tested in section 6 on an experimental test-bed as described in section 5. The results of tests of navigation are reported in section 7. Finally, the article ends with some concluding remarks and perspectives in section 8 .

\section{The sensor}

This section briefly describes the physical principles of the sensor. We refer the reader to [SJB $\left.{ }^{+} 11\right]$ for a more detailed description. Our first generation of sensors were named "slender probes" because of their high aspect ratio (length/thickness) shape. The sensor is composed of a body with insulating wetted boundaries on which are fixed a set of $m+1$ electrodes noted $\mathcal{E}_{0}, \mathcal{E}_{1} \ldots \mathcal{E}_{m}$ (Figure 2). The rear (tail) electrode $\mathcal{E}_{0}$ and front (head) $\mathcal{E}_{m}$ electrode, are both hemispherical while electrodes fixed on the body are ring shaped. The 2-electrode probe has $m=1$ and the 4-electrode probe has $m=3$. The voltage imposed on $\mathcal{E}_{\alpha=1,2 \ldots m}$ is the common ground while $\mathcal{E}_{0}$ is put under the controlled voltage $U$. Once the sensor immersed in a fluid, this active device produces a field of current lines in its surroundings flowing from $\mathcal{E}_{0}$ to the other electrodes $\mathcal{E}_{\alpha=1, \ldots, m}$. These current lines are closed through an electric circuit inside the sensor. This circuit measures the vector of the currents $\mathbf{I}=\left(I_{1}, I_{2}, \ldots, I_{m}\right)$ flowing across the $\mathcal{E}_{\alpha=1,2, \ldots, m}$. When an object is close to the sensor, it generates perturbations of the electric lines creating a portrait that is measured by the current measurement circuit. The entire device can be embarked on a mobile body such as an underwater motion controlled vehicle.
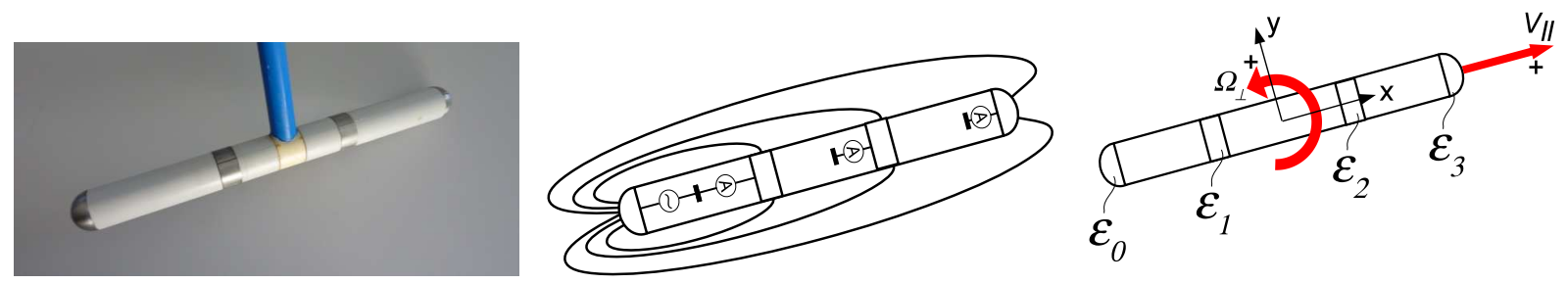

Figure 2: Picture of a slender probe (left), measurement scheme with electric field lines (center) and schematic view (right) of a 4-electrode sensor.

Based on these design, one can show $\left[\mathrm{SJB}^{+} 11\right]$, that for a given small aspect ratio $(\lesssim 0.1)$ of the sensor and a given sensitivity of its measurement electronics, the range of detection of a perturbative objet is a constant factor of the sensor length. For instance, in our case with a sensitivity of about $1 / 1000$ and an aspect ratio of $1 / 22$, the detection range of a wall is approximatively equal to the sensor length which corresponds to the range of mormyride fish. Finally, this range can be increased by increasing the length of the sensor (in fact the maximal length of a dipole supported by its body).

\section{Models}

Here we describe the model of measurement that relates the sensor measurements to its surroundings. 


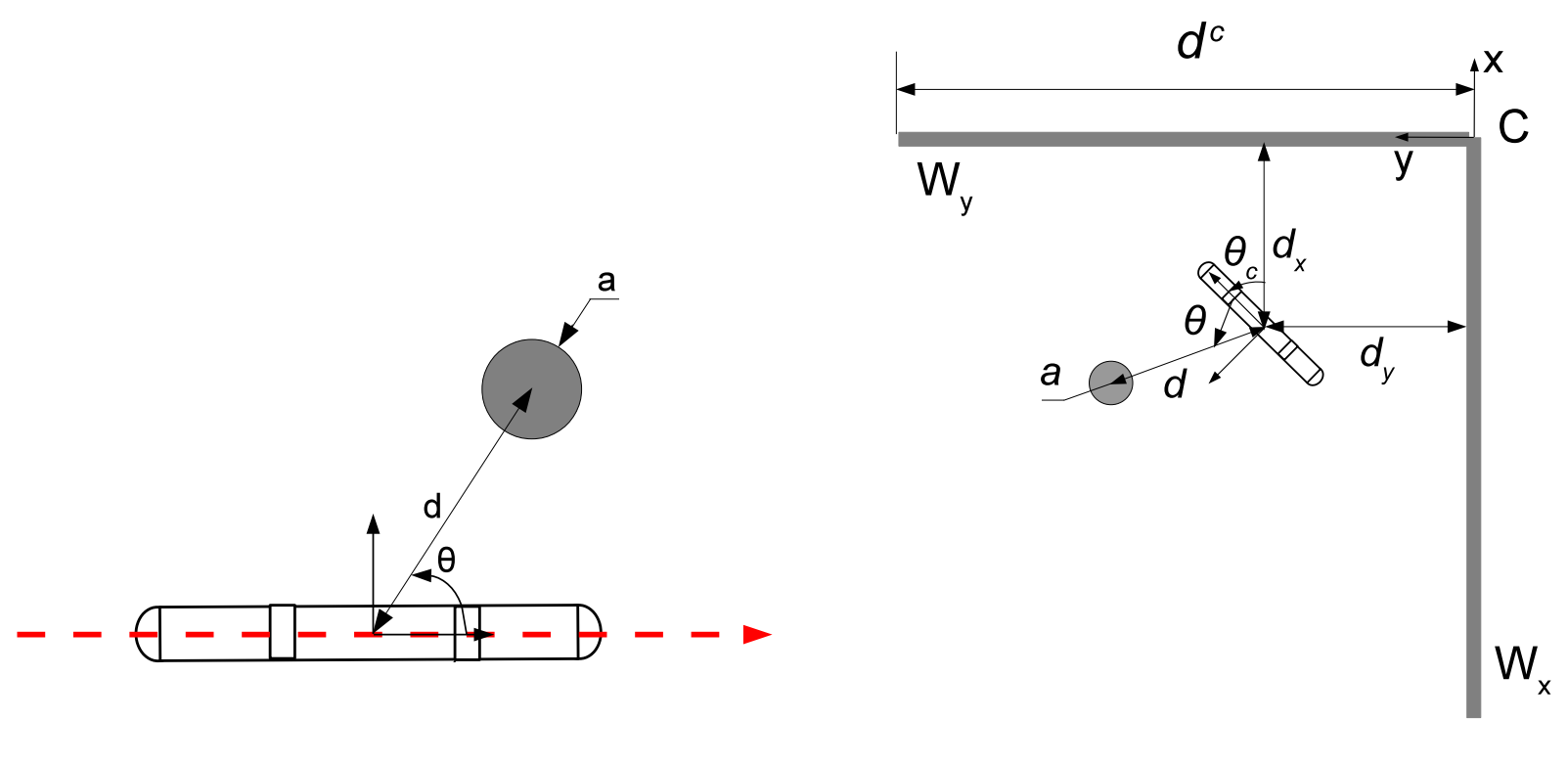

(a) Simple scene.

(b) Complex scene.

Figure 3: Parametrization of a simple (with only one object, here a sphere) and a complex scene (several objects, here a sphere and a corner). Note that $d^{c}=d_{A}^{c}\left(d_{A}^{c}\right.$ being the axial range of detection of a corner), if the sensor is parallel to the upper wall and $d^{c}=d_{L}^{c}$ (i.e. equal to the lateral range of detection of a corner) if it is perpendicular to the upper wall).

\subsection{Analytical model of measurement}

The sensor is modeled as an array of $m+1$ spherical conducting electrodes aligned on a rigid line that forms a solid axis [CHKN05]. In the following, the characteristics of the tested sensor are illustrated in Figure 2 with a radius $R=1 \mathrm{~cm}$, and total length $L=22 \mathrm{~cm}$. The conductivity of the water is $\gamma=0.04 \mathrm{~S} / \mathrm{m}$ (that of ordinary tap water). An electric field is produced around the sensor by imposing a voltage between one electrode $\mathcal{E}_{0}$ and all the others. This produces a field of current lines flowing across the measurement electrodes. The corresponding measured currents are influenced by the presence of objects in the sensor surroundings (Figure 3). An object placed in the environment will modify the electric field and the corresponding measured currents. The modeling of the measured currents can be accurately achieved by the boundary elements method [PLIB11], but here we are interested in analytical models that are easily computed onboard. We first evaluate the effect of a single small object (Figure 3(a)), and then, the combined effects of such an object in presence of an insulating corner (Figure 3(b)). In the latter, we resort to the "images modeling method" [Jak99]. The images modeling method is based on the use of imaginary charges placed outwith of the physical domain in order to emulate the boundary conditions of the problem. Although the modeling of spheres, walls and corners have been addressed in $\left[\mathrm{LCA}^{+} 10\right]$ and [BBG08] respectively (and separately), the case of scenes comprising spheres and walls as those studied later in our experiments has still not been considered. In all cases, the effects of the electrically contrasted objects on the sensor measurements can be modeled as an expression of the general form:

$$
\mathbf{U}=\mathbf{R}\left(\mathbf{x}, \gamma, \gamma_{s}\right) \mathbf{I}
$$

where $\mathbf{U}$ is the $m \times 1$ vector of the imposed voltages, $\mathbf{I}$ is the $m \times 1$ vector of the measured currents, $\mathbf{R}$ is the $(m \times m)$ resistance matrix of the scene, with $\mathbf{x}$, the state vector gathering all 
the geometric parameters of the scene, and $\gamma_{s}$ the electric conductivity of the sphere. For example, in Figure 3(a), the sphere location with respect to the sensor is parameterized by a distance $d$, an angle $\theta$ and the radius $a$. For this scene the state vector of geometric parameters is: $\mathbf{x}=(d, \theta, a)^{T}$. In all case, the measured currents of $\mathbf{I}$ can be expressed:

$$
\mathbf{I}=\mathbf{R}^{-1}\left(\mathbf{x}, \gamma, \gamma_{s}\right) \mathbf{U}
$$

which is highly non linear with respect to the state. We shall now consider the sensor moving in a cubic tank bounded by insulating walls ibetween which a small sphere has been placed. In these conditions, the resistance $\mathbf{R}$ offered to the sensor takes the most general form :

$$
\mathbf{R}=\mathbf{A}+\mathbf{S}+\mathbf{W}_{\mathbf{x}}+\mathbf{W}_{\mathbf{y}}+\mathbf{C},
$$

where

- A models the sensor's own resistance, which depends only on the geometry of the robot as detailed in $\left[\mathrm{JGB}^{+} 10, \mathrm{LCA}^{+} 10\right]$.

- $\mathbf{S}$ models the influence of the sphere which depends on $d, \theta, a, \gamma, \gamma_{s}$ and is based on a model initially proposed in [Ras96]. A further study of this influence is developed in $\left[\mathrm{LCA}^{+} 10\right]$.

- $\mathbf{W}_{x}, \mathbf{W}_{y}$ and $\mathbf{C}$ model the wall and the corner influence (Figure 3(b)) and depends on $d_{x}$ and $\theta_{c}\left(d_{y}\right.$ and $\left.\theta_{c}\right)$. This influence is studied in $\left[\mathrm{JGB}^{+} 10\right]$.

Finally, the model of measurements takes the general form:

$$
\mathbf{I}=\mathbf{h}\left(\mathbf{x}, \gamma, \gamma_{s}\right)
$$

For the sake of simplicity we will assume that: 1) the objects are motionless in the tank frame; 2 ) the sensor moves in its equatorial plane which is also a symmetry plane (top-bottom) for the objects; and 3) $\gamma$ and $\gamma_{s}$ are a priori known or measured [SJB $\left.{ }^{+} 11\right]$. The reconstruction thus only concerns the geometric state $\mathbf{x}$. It is in general difficult to estimate the scene's parameters because the model is non-linear and because of noise in the measurement. To overcome these difficulties, the sensor moves in order to enrich the number of measurements and the information they contain. In section 3.3, we present the model of displacement of the robot.

\subsection{Sensor's range}

The objective of this section is to determine the maximal distance at which the sensor can detect a perturbative object. When the range's measured along its axis $d_{A}$, its be named the axial range of object detection and when it is measured perpendicularly to the axis, it is named lateral range of object detection and denoted $d_{L}$ (see Figure $5(\mathrm{a})$ ). To the end, we define the following norm :

$$
I_{s}=\left\|\mathbf{I}-\mathbf{I}_{\infty}\right\|
$$

where $\mathbf{I}_{\infty}$ is the vector of currents measured in an unbounded environment (i.e. without objects, walls, or corners...), while $\mathbf{I}$ are the currents in the presence of objects. With our slender probes immersed in the test bench (tank), the currents induced by noise have approximately a norm $I_{b} \simeq 10^{-6} \mathrm{~A}$ (see $\left[\mathrm{SJB}^{+} 11\right]$ ). Thus, when the current $I_{s}>I_{b}$, the sensor detects an object in its surrounding. This threshold defines the range of the sensor beyond which it cannot perceive any change in its environment. In the following we study the sensor's range in presence of a sphere and of a corner. In the case of a sphere same simulations were carried out in $\left[\mathrm{LCA}^{+} 10\right]$, while those related to the corner have never been presented elsewhere. 


\subsubsection{Range for a small object}

The objective is to determine the distance at which the sensor detects a small object in an unbounded environment (see Figure 3(a)). To this end, we place a conductive sphere at different locations around the sensor within a $0.4 \mathrm{~m} \times 0.4 \mathrm{~m}$ area while avoiding collision with the sensor. The norm of the current $I_{s}$ associated with each position of the conductive sphere is computed for the 2-electrode (Figure 4(a)) and 4-electrode sensor (Figure 4(b)) using (5) with I given by (4) and $\mathbf{R}=\mathbf{A}+\mathbf{S}$ in (3). In Figure 4(a), we clearly observe a "blind spot" at the middle of the 2-electrode sensor. This characteristic is absent with the 4-electrode sensor Figure 4(b). This feature is observed when the distance between any pair of electrodes is much higher than the size $a$ of the object. Thus, for any given length $L$, the area enclosed by a level contour $I_{s}\left(=I_{b}\right)$ of the 4-electrode sensor is wider than that of the 2-electrode sensor. Figure 5(b) shows the axial $d_{A}^{s}$ and lateral $d_{L}^{s}$ ranges of the 2-electrode and 4-electrode sensors for several values of radius $(a=[0.01,0.03,0.05,0.10] \mathrm{m})$ of a conductive sphere (the upper index $s$ means "sphere"). The axial range $d_{A}^{s}$, is similar for both sensors, and for the 4-electrode sensor, $d_{L}^{s}$ and $d_{A}^{s}$ are also very similar. With the 2-electrode sensor, the sensor can detect a sphere of radius $a \leq 0.01 \mathrm{~m}$ laterally, only when this object is close to one of the electrodes. On the other hand, along the body of the 4-electrode sensor, such a small object can be always detected.

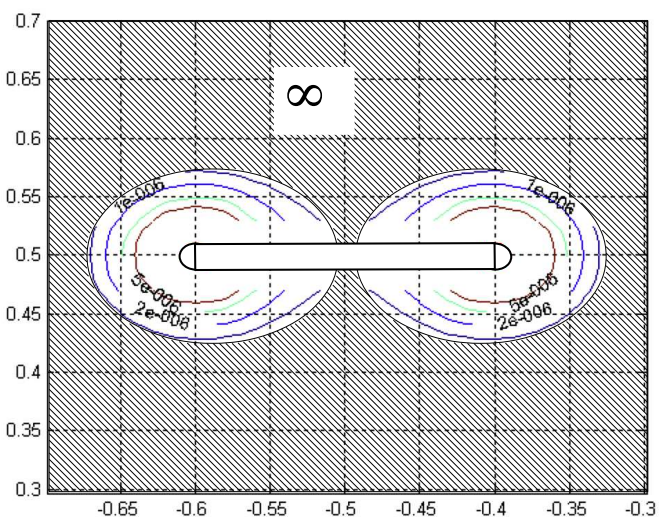

(a) The contours of the iso-current $I_{s}$ for the 2electrode sensor.

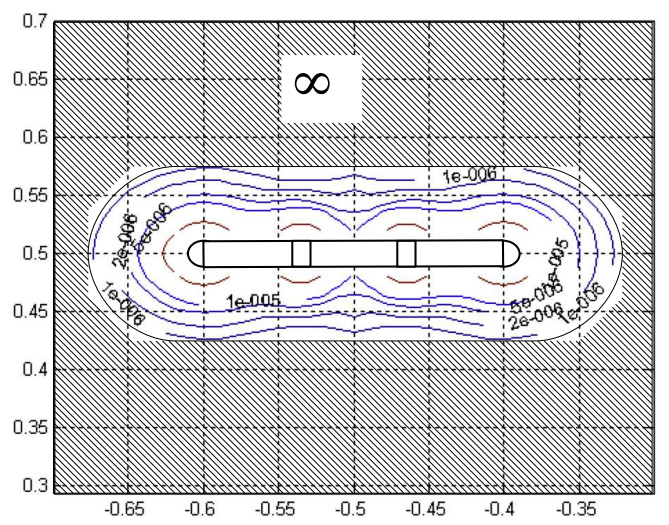

(b) The contours of the iso-current $I_{s}$ for the 4electrode sensor.

Figure 4: The contours of the iso-current $I_{s}$ for a sphere of radius $0.01 \mathrm{~m}$. The sensor cannot detect objects in the hatched part. 


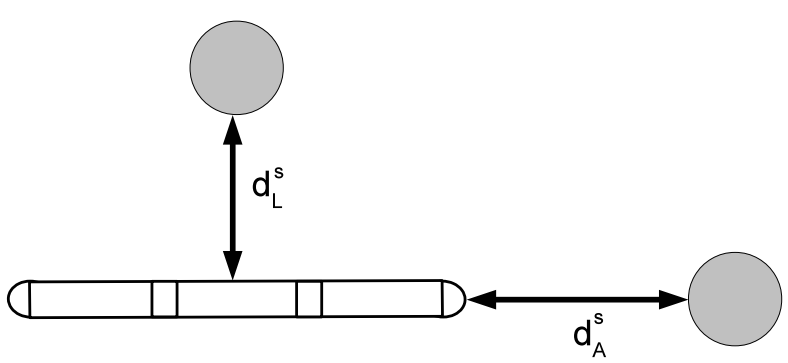

(a) Illustration of the axial and lateral ranges of detection for a sphere. Beyond $d_{L}^{s}$ and $d_{A}^{s}$, the sensor cannot detect the presence of the sphere.

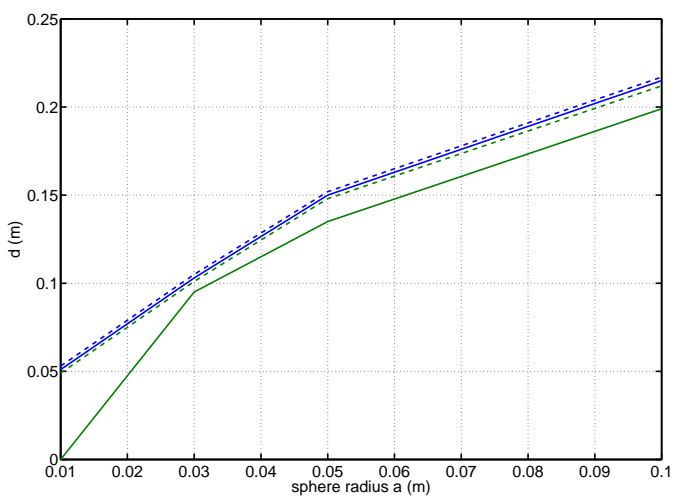

(b) Ranges $\left(d_{A}^{s}\right.$ (blue) and $d_{L}^{s}$ (green) of the 2 (bold line) and 4 (dotted line) -electrode sensor) for several values of the sphere radius.

Figure 5: Axial and lateral ranges of detection of a sphere.

\subsubsection{Sensor's range in a corner}

The objective is to determine the distance at which the 4-electrode sensor detects a corner (see Figure 3(b)). We put the 4-electrode sensor at different locations at an orientation $\alpha$ to the corner and compute (5) with $\mathbf{I}$ given by (4) and $\mathbf{R}=\mathbf{A}+\mathbf{W}_{\mathbf{x}}+\mathbf{W}_{\mathbf{y}}+\mathbf{C}$, in (3). Figures 6(a) and 6(b) display the current $I_{s}$ for orientations of $\alpha=0^{\circ}$ and $\alpha=45^{\circ}$. When the sensor orientation changes, the iso-current plots shift from those of Figure 6(a) to those of Figure 6(b). Note that when the sensor moves towards the corner in parallel to one of the walls $\left(\alpha=0^{\circ}\right)$, it perceives the front wall better than the lateral wall $\left(d_{A}^{c}>d_{L}^{c}\right)$. When the sensor tilts, the influence of the corner increases. Quantitatively, when the sensor follows the wall in parallel, the detection range of a corner is about $d_{A}^{c} \approx 0.65 \mathrm{~m} \approx 3$ times the sensor's length (i.e. $d_{A}^{c} \simeq 3 L=3 \times 22 \mathrm{~cm}$ ).

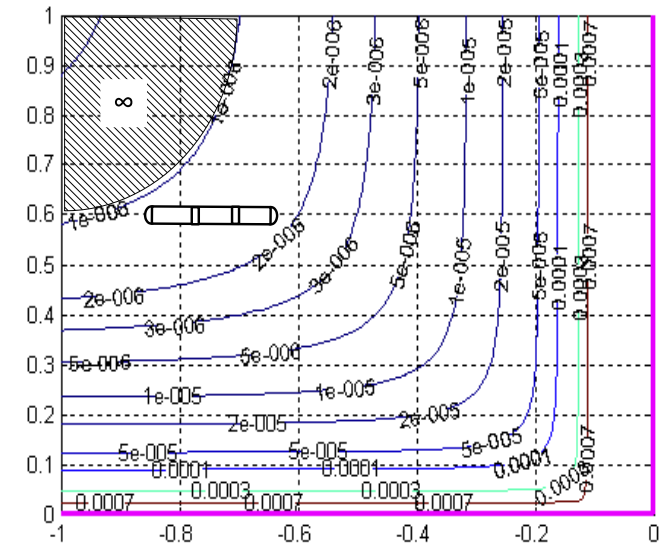

(a) The contours of the iso-current $I_{s}$ for a sensor tilted of $\alpha=0^{\circ}$.

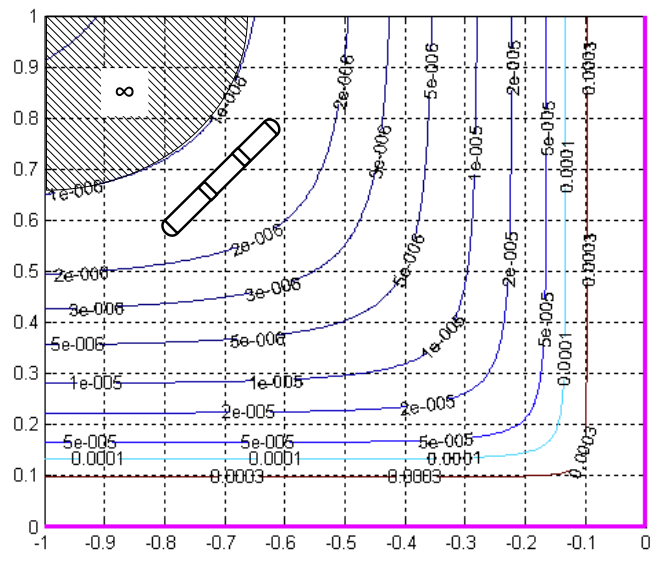

(b) The contours of the iso-current $I_{s}$ for a sensor tilted of $\alpha=45^{\circ}$.

Figure 6: The contours of the current $I_{s}$ for the sensor close to a corner. When the sensor is in the hatched part bounded by the iso-current $I_{s}=I_{b}$, it cannot detect the corner. 


\subsection{Robot displacement model}

The sensor motion is modeled as that of a non-holonomic unicycle whose motion parallel to the lateral $y$ body axis is constrained to zero (Figure 2). The velocities $V_{\|}$(axial velocity, i.e. along the $x$ body axis) and $\Omega_{\perp}$ (yawing velocity) define the vector of control inputs $\mathbf{u}=\left(V_{\|}, \Omega_{\perp}\right)^{T}$. In the scene of Figure 3(b), the sphere and the corner (defined by the walls $W_{x}, W_{y}$ and their intersection $C$ ) are fixed in space, so the model of displacement of the scene with respect to the sensor can be expressed in the discrete form:

$$
\begin{aligned}
& \text {-Displacement model of the corner: }\left\{\begin{aligned}
d_{x}^{k+1} & =d_{x}^{k}-V_{\|}^{k} \cos \left(\theta_{c}^{k}\right) \Delta t, \\
d_{y}^{k+1} & =d_{y}^{k}-V_{\|}^{k} \sin \left(\theta_{c}^{k}\right) \Delta t, \\
\theta_{c}^{k+1} & =\theta_{c}^{k}+\Omega_{\perp}^{k} \Delta t,
\end{aligned}\right. \\
& \text {-Displacement model of the sphere: }\left\{\begin{aligned}
d^{k+1} & =d^{k}-V_{\|}^{k} \cos \left(\theta^{k}\right) \Delta t, \\
\theta^{k+1} & =\theta^{k}+\left(\Omega_{\perp}^{k}+\frac{V_{\|}^{k} \sin \left(\theta^{k}\right)}{d^{k}}\right) \Delta t, \\
a^{k+1} & =a^{k}
\end{aligned}\right.
\end{aligned}
$$

where $\Delta t$ is the sampling period, and $k$ denotes the time step at which the vector of geometric variables is computed as $\mathbf{x}^{k}=\left(d_{x}^{k}, d_{y}^{k}, \theta_{c}^{k}, d^{k}, \theta^{k}, a^{k}\right)^{T}$. Finally, the displacement model can be written in the general form :

$$
\mathbf{x}^{k+1}=\mathbf{f}\left(\mathbf{x}^{k}, \mathbf{u}^{k}\right),
$$

where $\mathbf{x}^{k}$ is the geometric state vector of the scene and the vector of control inputs is $\mathbf{u}^{k}=$ $\left(V_{\|}^{k}, \Omega_{\perp}^{k}\right)^{T}$.

\section{Kalman Filter}

The objective of the observer is to estimate the geometric parameters of the environment gathered into the (geometric) state vector $\mathbf{x}$. The chosen observer is an unscented Kalman filter [SJ97]. This choice has the advantages of the classical extanded Kalman filter [Kal60, NdS90] while avoiding the complex analytical linearization of the displacement equations (7) and measurement model (4). As with any Kalman filter, our filter requires the definition of some matrices which tune its dynamic features. The first is the covariance matrix $\mathbf{P}_{k}$ which is related to the state variables $\mathbf{x}^{k}$ and represents a trust indicator associated with the estimated state, a small covariance meaning that the state is precisely known, and a large covariance that it is imprecisely known. The evolution of $\mathbf{P}_{k}$ is defined by the unscented Kalman filter algorithm. Two other matrices are required: $\mathbf{Q}_{m}$, which defines whether the model (7) is well known or not, and $\mathbf{Q}_{s}$, which determines the level of noise in the measurements (4). The principle of the unscented Kalman filter is reminded in Figure 7. Based on its covariance $\mathbf{P}_{k}$, the filter computes some sampling points (called "sigma points" $\chi^{k+1}$ ) around the estimated state $\hat{\mathbf{x}}^{k}$ using a deterministic sampling technique known as the unscented transform. It then propagates the sigma points $\chi$ according to: 1) the evolution model $\boldsymbol{\chi}^{k+1}=\mathbf{f}\left(\boldsymbol{\chi}^{k}, \mathbf{u}^{k}\right)$; and 2) the model of measurements $\tilde{\mathbf{y}}=\mathbf{h}\left(\boldsymbol{\chi}^{k}, \gamma, \gamma_{s}\right)$, which improves the convergence of the estimated state $\hat{\mathbf{x}}^{k+1}$ and the covariance $\mathbf{P}_{k+1}$. 


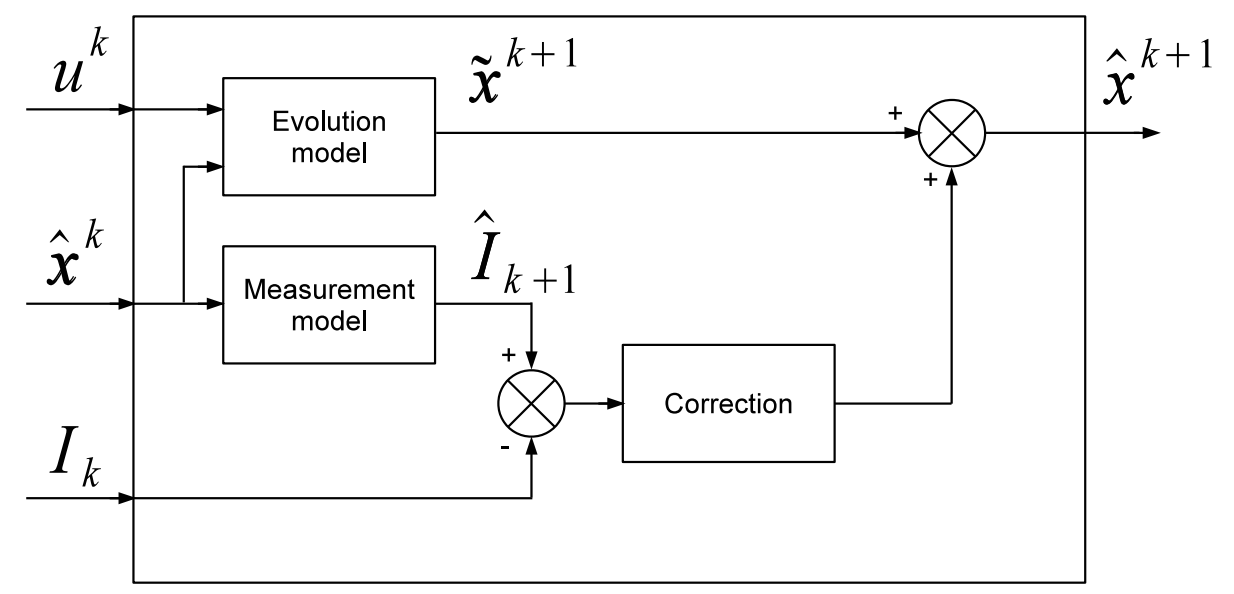

Figure 7: Chart flow illustrating the principle of the unscented Kalman filter.

The equations of the unscented Kalman filter are summarized in the following set of recursive equations: in step $k$, the estimated state is $\hat{\mathbf{x}}^{k}$ with covariance matrix $\mathbf{P}_{k}$. In the first step, this the filter computes the "sigma points" $\chi^{k+1}$ around the estimated state $\hat{\mathbf{x}}^{k}$ using the unscented transform:

$$
\left\{\begin{array}{l}
\chi_{0}^{k}=\hat{\mathbf{x}}^{k} \\
\chi_{i}^{k}=\hat{\mathbf{x}}^{k}+\sqrt{(N+\kappa)\left(\mathbf{P}_{k}+\mathbf{Q}_{m}\right)} \text { for } i=1 \text { to } N \\
\chi_{i}^{k}=\hat{\mathbf{x}}^{k}-\sqrt{(N+\kappa)\left(\mathbf{P}_{k}+\mathbf{Q}_{m}\right)} \text { for } i=N+1 \text { to } 2 N,
\end{array}\right.
$$

where $N$ is the size of the state vector and $\kappa$ is a parameter that must be chosen $(\kappa=1)$. These sigma $\chi_{i}^{k}$ points are weighted using weight coefficients:

$$
W_{0}=\frac{\kappa}{\kappa+N}, W_{i}=\frac{1}{2(\kappa+N)}
$$

In a second step the filter propagates the sigma points $\chi$ according to:

- The evolution model:

$$
\begin{aligned}
\tilde{\chi}_{i}^{k+1} & =\mathbf{f}\left(\chi_{i}^{k}, \mathbf{u}_{k}\right), i=0, \ldots, 2 N \\
\tilde{\mathbf{x}}^{k+1} & =\sum_{i=0}^{2 N}\left(W_{i} \tilde{\boldsymbol{\chi}}_{i}^{k+1}\right), \\
\tilde{\mathbf{P}}_{k+1} & =\sum_{i=0}^{2 N}\left(W_{i}\left(\tilde{\chi}_{i}^{k+1}-\tilde{\mathbf{x}}^{k+1}\right)^{T}\left(\tilde{\boldsymbol{\chi}}_{i}^{k+1}-\hat{\mathbf{x}}^{k+1}\right)\right),
\end{aligned}
$$

where $\tilde{\boldsymbol{\chi}}_{i}^{k+1}$ is the estimated value of $\boldsymbol{\chi}_{i}^{k}, \mathbf{u}_{k}$ is the input control, $\tilde{\mathbf{x}}^{k+1}$ is the state prediction and $\tilde{\mathbf{P}}_{k+1}$ is the predicted covariance.

- The model of measurements, which is given by:

$$
\begin{aligned}
& \tilde{\mathbf{y}}_{\mathbf{i}}^{k+1}=h\left(\tilde{\boldsymbol{\chi}}_{i}^{k+1}, \gamma, \gamma_{s}\right), i=0, \ldots, 2 N, \\
& \hat{\mathbf{I}}_{k+1}=\sum_{i=0}^{2 N} W_{i} \tilde{\mathbf{y}}_{\mathbf{i}}^{k+1},
\end{aligned}
$$

where $\tilde{\mathbf{y}}_{\mathbf{i}}^{k+1}$ is the measurement prediction and $\hat{\mathbf{I}}_{k+1}$ is the estimated measurement. 
- A correction on the estimated state:

$$
\begin{aligned}
& \hat{\mathbf{x}}^{k+1}=\tilde{\mathbf{x}}^{k+1}+\mathbf{K}_{k+1}\left(\mathbf{I}_{k}-\hat{\mathbf{I}}_{k+1}\right), \\
& \mathbf{P}_{k+1}=\tilde{\mathbf{P}}_{k+1}-\mathbf{K}_{k+1} \mathbf{P}_{\mathbf{y y}} \mathbf{K}_{k+1}^{T},
\end{aligned}
$$

where

$$
\begin{aligned}
& \mathbf{K}_{k+1}=\mathbf{P}_{\mathbf{x y}} \mathbf{P}_{\mathbf{y y}}{ }^{-1} \\
& \mathbf{P}_{\mathbf{y y}}=\sum_{i=0}^{2 N}\left(W_{i}\left(\tilde{\mathbf{y}}_{\mathbf{i}}^{k+1}-\hat{\mathbf{I}}_{k+1}\right)^{T}\left(\tilde{\mathbf{y}}_{\mathbf{i}}^{\mathbf{k}+\mathbf{1}}-\hat{\mathbf{I}}_{\mathbf{k}+\mathbf{1}}\right)\right)+\mathbf{Q}_{\mathbf{s}}, \\
& \mathbf{P}_{\mathbf{x y}}=\sum_{i=0}^{2 N}\left(W_{i}\left(\tilde{\boldsymbol{\chi}}_{\boldsymbol{i}}^{k+1}-\tilde{\mathbf{x}}^{k+1}\right)^{T}\left(\tilde{\mathbf{y}}_{\mathbf{i}}^{\mathbf{k}+\mathbf{1}}-\hat{\mathbf{I}}_{\mathbf{k}+\mathbf{1}}\right)\right),
\end{aligned}
$$

$\hat{\mathbf{x}}_{k+1}$ is the estimated state of $\mathbf{x}_{k+1}$ and $\mathbf{P}_{k+1}$ is the estimated covariance.

\section{$5 \quad$ Experimental setup}

We now report some experimental results which illustrate the filter performances. Section 5.1 describes the test bench. We first assess the accuracy of the model used by the filter (4), and then introduce the experiments based on the filter.

\subsection{Tank and cartesian robot}

An automated test bench consisting in a tank of one cubic meter volume and a three-axis cartesian robot has been built to test our electrolocation sensors and algorithms in controlled and repeatable conditions (see Figure 8). The robot is fixed on top of the aquarium and allows the probes to be positioned in translation along $X$ and $Y$ with a precision of $1 / 10 \mathrm{~mm}$. The maximum speed is $300 \mathrm{~mm} / \mathrm{s}(\simeq 1 \mathrm{~km} / \mathrm{h})$ for both translations. Orientation in the $(X, Y)$ plane is adjusted in increments of $0.023^{\circ}$ using an absolute yaw-rotation stage whose maximum velocity is $80^{\circ} / \mathrm{s}$ $(13.5 \mathrm{tr} / \mathrm{min})$. The motion of the three axis robot is controlled using simulink software with the dSpace system. The probes are positioned in the aquarium at adjustable height $(Z)$ using a rigid glass epoxy fibre tube. This vertical insulating tube forms a conduit for the cables conveying the signal from the probe electrodes. The electrolocation signals are first processed by an analogue chain (amplification and filtering) then digitally converted using a 16 bit ADC (DS2004) card with a resolution of $0.3 \mathrm{mV} /$ bit (range $+/-10 \mathrm{~V}$ ). 


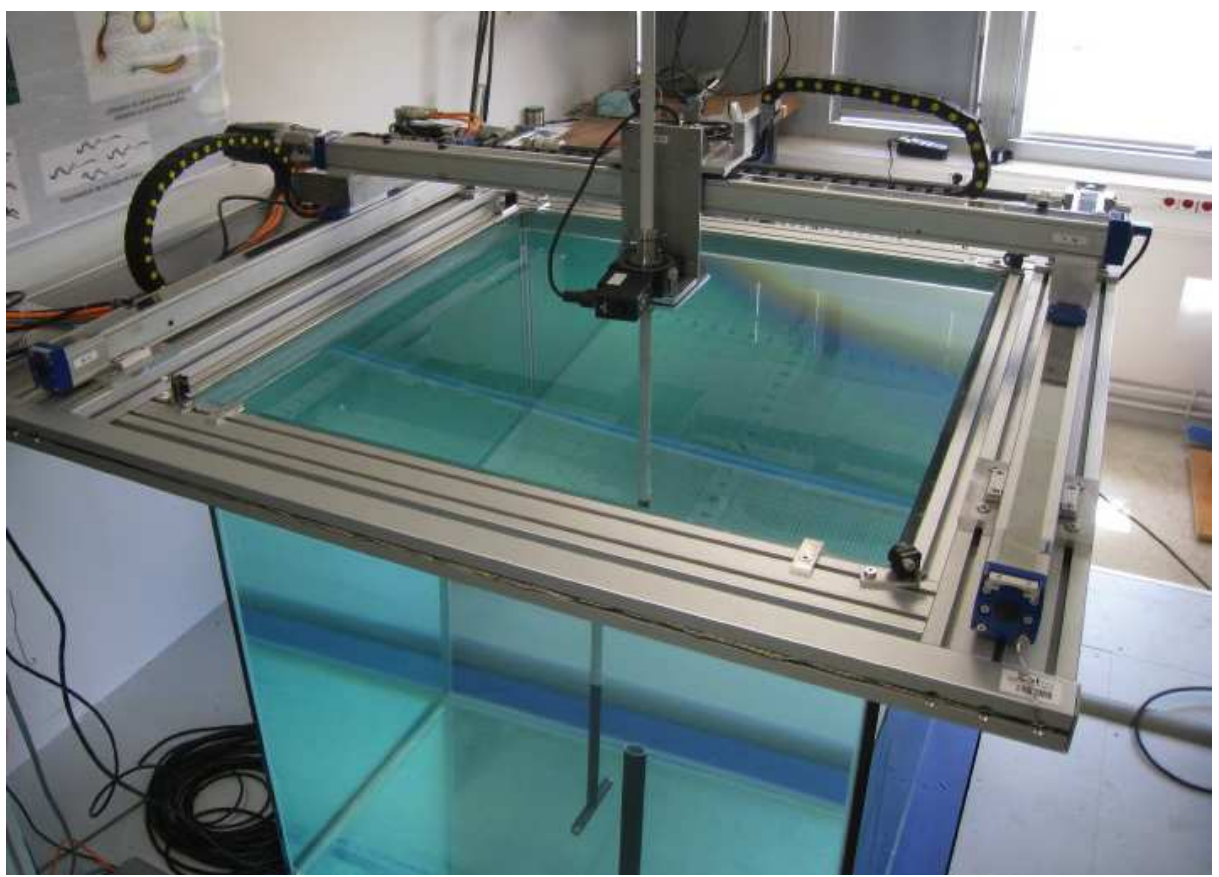

Figure 8: Electrolocation test bench: the sensor is attached to the tip of a stick whose planar motions are controlled with a Cartesian robot fixed on the top of the tank.

\subsection{Validation of the current model}

We first compare the measured and the modeled currents for the 2 and 4-electrode sensors. Two validation tests are carried out. The first validates the model of the empty tank, while the second assesses the model of a sphere in an unbounded environment. Note that the simulation of a slender probe in presence of a sphere has been done in $\left[\mathrm{LCA}^{+} 10\right]$ with the same sensor and in $\left[\mathrm{BGJ}^{+} 12\right]$ for a more complex sensor including a left-right partition of the electrodes (i.e. with a kind of "binocular" sensor). Likewise, the simulation of the sensor with walls has been done in [BBG08] and with corners in $\left[\mathrm{JGB}^{+} 10\right]$.

\subsubsection{Current model of the tank}

The first test consists in moving the sensor $\left(V_{\|}=0.04 \mathrm{~m} / \mathrm{s}\right)$ in the tank at a constant distance $\left(d^{d}=\right.$ $15 \mathrm{~cm}$ ) from the walls (see Figure 3(b)) and comparing the measured currents with those calculated by the model (4), with the actual state $\mathbf{x}$, and with the actual conductivity $\gamma$. Figure 9 (a) shows the modeled and measured currents for the 2 and 4-electrode sensors. The modeled currents show a discontinuities (points A in Figure 10) as well as some discrepancies with experimental results. This is probably due to the fact that the global model of the sensor in the tank (as given by a boundary elements numerical code for example) is in our case replaced by the local models of the four corners between which commutations are forced (at points $A$ ). This choice has been adopted for several reasons: $1^{\circ}$ ) the sensor has a local perception of the corners bounded by $d_{A}^{c}$ (of section 3.2). $2^{\circ}$ ) A global analytical model would require to define an infinite number of images (like two mirrors positioned face to face), what would cause problems of convergence of the method of images. $3^{\circ}$ ) At last, in the perspective of using this model for observation, local models will allow us to navigate without à priori knowledge of the size of the tank. The details of how the commutations are managed will be treated in section 7 when dealing with navigation. Figure $9(\mathrm{~b})$ 
shows the 3 measured currents of the sensor (red) as well as the model calculated currents (black), when the 4-electrode sensor follows the walls of the tank. The results show that the measured and calculated currents are sufficiently close to each other in order to consider the model good enough to be used for the reconstruction and navigation task.

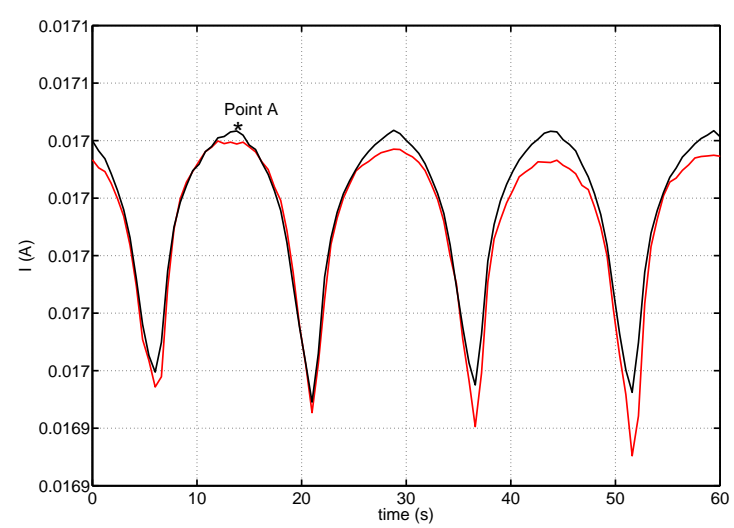

(a) Comparison between measured and calculated currents with a 2-electrode probe.
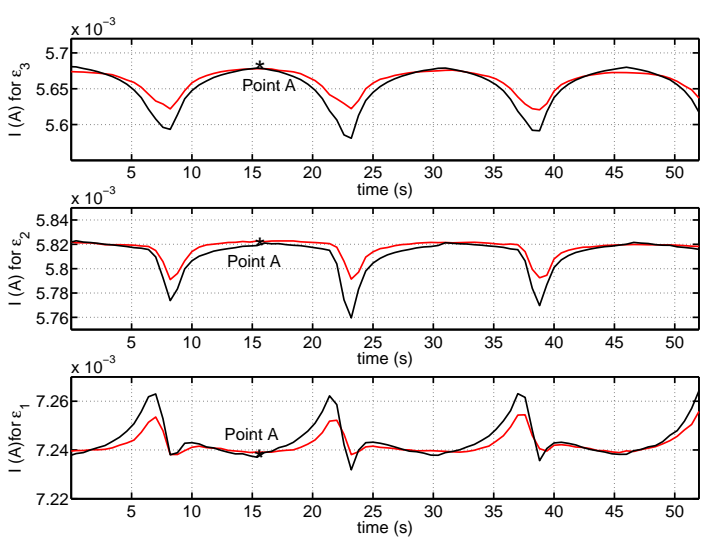

(b) Comparison between measured and calculated currents with a 4-electrode probe.

Figure 9: Measured (red) and modeled currents (black) with the real state vector $\mathbf{x}$ when the sensors follow the walls of the tank $\left(V_{\|}=0.04 \mathrm{~m} / \mathrm{s}\right.$ and $\left.d^{d}=15 \mathrm{~cm}\right)$ (see also Figure 14).

\subsubsection{Current model of the sphere}

In the following, a small object (sphere, cube) in the surroundings of the sensor is modeled as a sphere whose the size and location (related to the sensor) have to be reconstructed by the filter. Before testing the sphere localization algorithm, two preliminary tests were carried out in order to assess whether the modeling assumptions which will model any object as its encapsulating sphere, are justified.
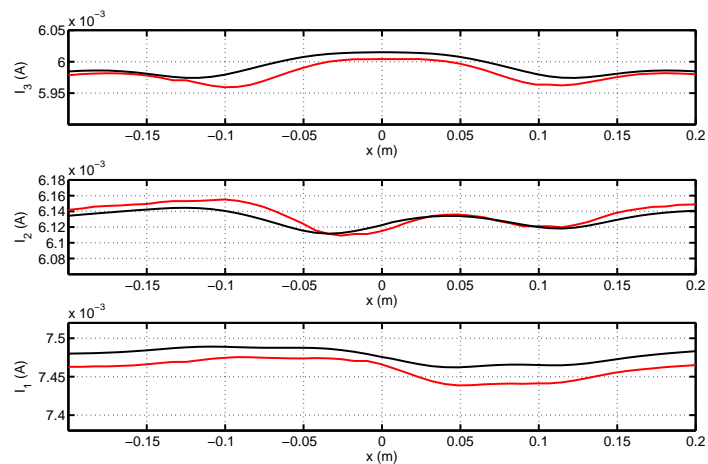

(a) The probe approaches an insulating sphere.

Figure 10: Measured (red) and modeled currents (black) with the real state vector $\mathbf{x}$ when the probe approaches a small object (sphere model).
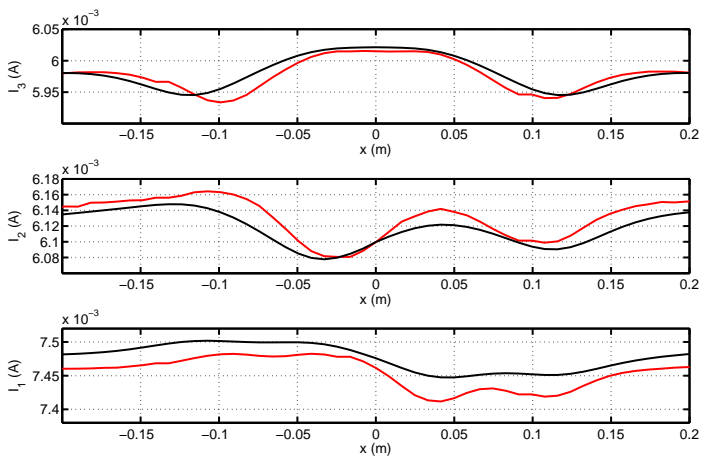

(b) The probe approaches a insulating cube. 
All these tests were carried out by comparing the actual measurements with those given by the model (4), once the model has been informed of the actual geometric (and electric) parameters of the scene. In the first test, a sphere is placed in the middle of the tank. In this location the electric effect of the walls are small, and the measurements are recorded in the conditions of Figure 3(a). The measurements are then computed using the model (4) with the actual extended state $\left(\mathbf{x}, \gamma, \gamma_{s}\right)$, and for a scene approximated by a sphere immersed in an unbounded fluid domain. The results show that the measured and calculated currents are sufficiently close to each other in order to consider the model of the sphere as enough accurate for our filter. These differences have essentially two origins. Firstly, the model of the sphere assumes that its size is small with respect to the spatial variations of the basal electric field produced by the sensor, a condition which is not satisfied here (the diameter of the sphere is about $6 \mathrm{~cm}$, i.e. of the order of the length of the sensor). Secondly, the insulating parts of the sensor are approximated as in [JGB $\left.{ }^{+} 10\right]$ (we refer the reader to $\left[\mathrm{BGJ}^{+} 12\right]$ for a more accurate model of the sensor). In the second test, the measurements are carried out with a cube located in the middle of the tank, and compared with the same model as that used in the previous test (a single sphere in an unbounded environment). Here, the sensor moves in a straight line with $V_{\|}=0.04 \mathrm{~m} / \mathrm{s}$ and $\Omega_{\perp}=0$. The Figures $10(\mathrm{a})-10(\mathrm{~b})$ show the 3 measured currents of the sensor (red) as well as the model calculated currents (black), when the probe approaches the object. The results in Figure 10(a), were produced using an insulating sphere of a radius $a=0.0305 \mathrm{~m}$. The results show that the measured and calculated currents are sufficiently similar to consider the model of the sphere good enough for its reconstruction. The plots in Figure 10(b) were obtained using a real insulating cube (of side-length $a=0.040 \mathrm{~m}$ ) located in the middle of the tank, and with the model of a sphere of radius $a=0.037 \mathrm{~m}$. The measured and calculated currents are enough close to each other, in order to conclude that the approximation of a cube by an equivalent sphere is justified. Going further into the details, let us point out that the peaks of the measured currents (for both the sphere and the cube) coincide well with those given by the sphere model. Thus, since each of these extrema corresponds to a configuration where one electrode is in font of the object center, they are directly related to the localization of the real objects and its spherical model in the sensor frame. As a result, the approximation of a compact object (as the cube) by a sphere should not compromise the localization of the object. Physically, this can be explained by the fact that the multipolar expansion (on a basis of spherical harmonics for instance) of the cube and the sphere responses to an external field can be only distinguished at a high order of expansion, while localization essentially requires the lowest order contributions. Finally, these properties will be confirmed and exploited in section 6 when we will address the electrolocation of small objects.

\section{Electro-localization}

Three experiments were carried out in our test bench to calibrate the performance of the algorithms of object reconstruction and localization. Two used the conditions illustrated in Figure 3(a), first with a small insulating sphere, and second, with a small insulating cube. In both experiments, the object was modeled by an equivalent sphere whose size and location are estimated. The third experiment used the scenario of Figure 3(b), where an insulating sphere is placed in a corner while the robot follows the walls of the tank. All these experiments were carried out with the 4-electrode sensor. The third experiment focussed on the ability of the algorithm to extract the parameters of a small object, immersed in a complex scene. For each of the experiments, we recorded the velocity $\left(V_{\|}\right.$and $\left.\Omega_{\perp}\right)$ and the measured currents $\mathbf{I}=\left(I_{1}, I_{2}, I_{3}\right)^{T}$. 


\subsection{Reconstruction of a sphere in a simple scene (fig. 3(a))}

By "simple scene", we mean that there is only one small object (here a sphere) in the sensor surroundings. In this first test devoted to the (geometric) state reconstruction, the robot moves forward in the tank in which an insulating sphere with a radius $a$ has been immersed. The real and estimated initial states of the scene are defined by: $(d=0.257 \mathrm{~m}, \theta=0.294 \mathrm{rad}, a=0.0305$ $\mathrm{m})$ and: $\hat{d}=d-0.1 \mathrm{~m}, \hat{\theta}=\theta+0.2 \mathrm{rad}, \hat{a}=a-0.02 \mathrm{~m})$. Figure 11(a) shows the change over time of the real (red) and estimated (blue) states of the sphere. Figure 11(b) represents the real (cyan) and the estimated (blue) scenes over time. When the object is close to the head (first electrode, at time $t=2 \mathrm{~s}$ ), the sphere is quite well localized but its estimated size is higher than its actual size. When it is located between the head and the tail $(2 \mathrm{~s}<t<8 \mathrm{~s})$, the estimated size of the sphere is close to its actual size. When the object is close to the emitter $(t=8 \mathrm{~s})$, the real sphere (its location and size) is again well reconstructed with a small estimation error. Finally, let us remark that in all experiments, "reconstruction" reaches its highest level of accuracy when the probe overtakes the object of interest and leaves it behind its back. This is due to the fact that the last electrode being the emitter, when the object is close to it, all the receivers are strongly perturbed by the object so increasing the information content of the measurement, compared to the receivers which are excited one by one. Furthermore, the emitter passing close to the object at the end, it benefits from the work of all the receivers and achieve the localization.
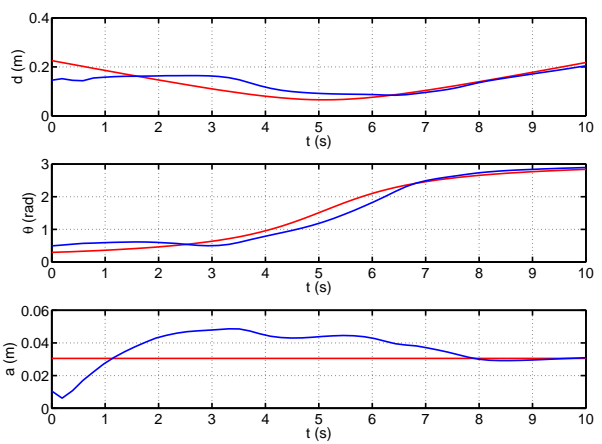

(a) Real (red) and estimated (blue) state of the sphere.

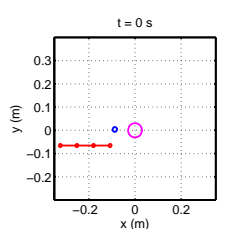

$\mathrm{t}=6 \mathrm{~s}$
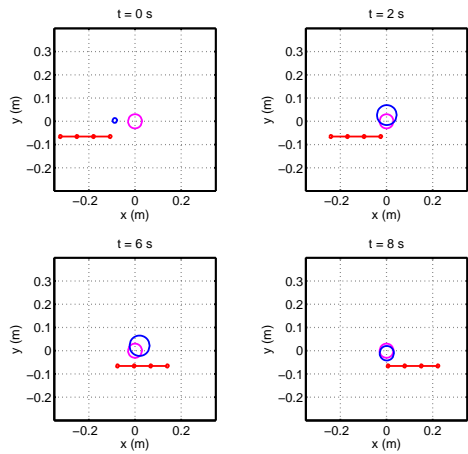

$t=8 \mathrm{~s}$
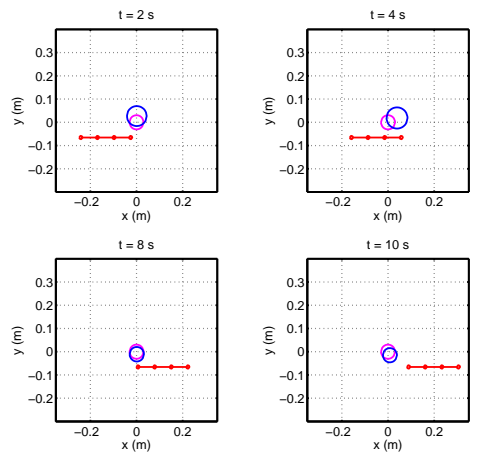

$t=10 \mathrm{~s}$

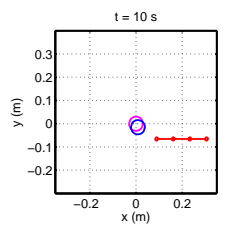

(b) Real (cyan) and the estimated (blue) scene.

Figure 11: Reconstruction of a sphere in a simple scene $\left(V_{\|}=0.04 \mathrm{~m} / \mathrm{s}\right)$. The state of the sphere is $(d, \theta, a)$.

Since the size and the location of the reconstructed object converge toward their real values, this experiment shows that thanks to the sensor motion, the filter can solve the ambiguity between size and distance and can distinguish a small object at short distance from a bigger one at a larger distance. However, the electric parameters of the scene being known a priori by the filter, the other ambiguity between conductivity and size $^{1}$ is still an open problem for the approach.

\footnotetext{
${ }^{1}$ The perturbed currents produced by a small object contain a factor including the size times a function of the electric conductance of the object and water named contrast factor.
} 


\subsection{Reconstruction of a cube in a simple scene (Figure 3(a))}

This second test is carried out in the same conditions as the first, except that the object is now an insulating cube. The filter estimates the parameters of an equivalent sphere using the sphere model. The initial real and estimated states of the scene are set to be: $(d=0.225 \mathrm{~m}, \theta=0.298$ $\mathrm{rad}, a=0.037 \mathrm{~m}$ (equivalent radius)) and: $(\hat{d}=d-0.1 \mathrm{~m}, \hat{\theta}=\theta+0.2 \mathrm{rad}, \hat{a}=0.001 \mathrm{~m})$. Figure 12(a) shows the change over time of the real (red) and estimated (blue) state of the cube. Figure 12(b) represents the actual (cyan) and the estimated (blue) scenes at different times of the sensor motion. As in the previous test, when the object is close to the head $(t=2 \mathrm{~s})$, the cube is quite well localized but its estimated size is greater than the actual length of the side of the cube. When the object is close to the emitter $(t=8 \mathrm{~s})$, its equivalent sphere is well reconstructed and the estimated error (on size and location) is small. This test illustrates a common feature of all the tests carried out: the estimated equivalent sphere always encapsulates the cube, and its location is well estimated. This confirms the prediction of section 5.2 and is due to the fact that when excited by an external field (here the basal field emitted by the sensor), any small compact object appears (from the point of view of the electric measurements of the sensor) as a polarized sphere at the leading order.
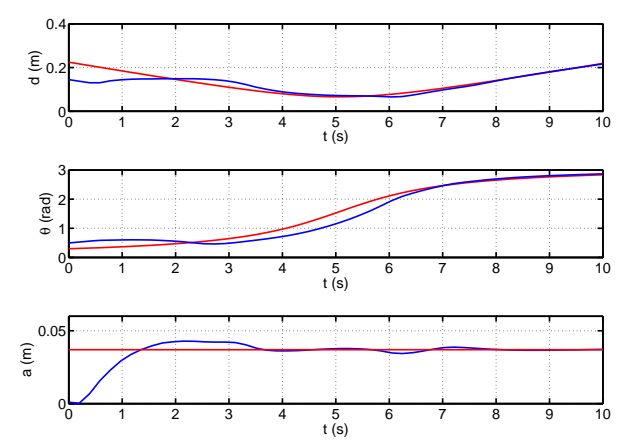

(a) Real (red) and estimated (blue) state of the cube.
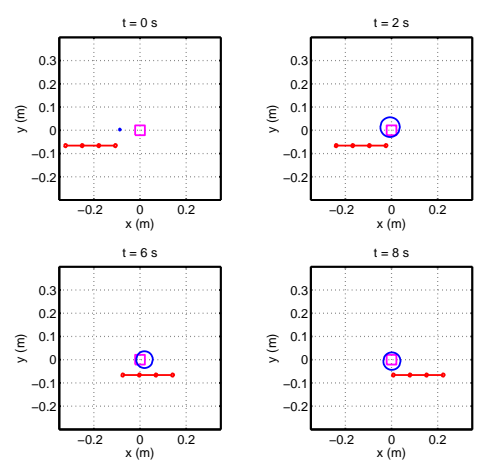

$\mathrm{t}=8 \mathrm{~s}$
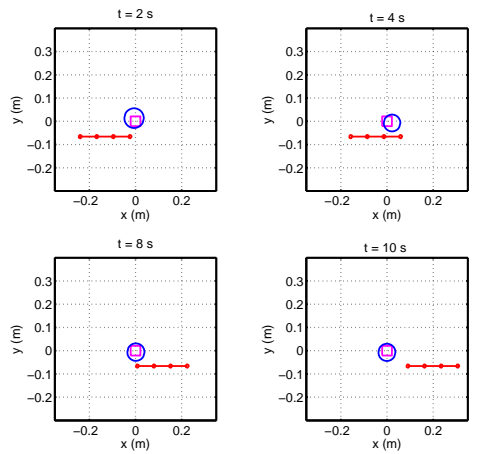

(b) Real (cyan) and the estimated (blue) scene.

Figure 12: Reconstruction of a cube in a simple scene $\left(V_{\|}=0.04 \mathrm{~m} / \mathrm{s}\right)$ using the model of a sphere: the cube is encapsulated in an equivalent sphere of state $(d, \theta, a)$

In nature, electric fish are able to distinguish between different shaped objects of close multipolar signatures (typically a cube and a sphere) [vdESG $\left.{ }^{+} 98\right]$. For instance, this can be achieved by exploring more actively the object by using motions of "va et vient" or alternately turning the head and the tail towards the object. In this case, we could explore the sharp edges of the cube where electric charges are accumulated by virtue of the peak effect. However, this article being focused on localization and not on shape recognition, its objective is rather to show that any small compact object can be encapsulated in an equivalent sphere with comparable size and location.

\subsection{Localization and reconstruction of a sphere in a complex scene.}

This test is carried out using the conditions illustrated in Figure 3(b), with an insulating sphere located in a corner while the robot follows the wall. This test focuses on the ability of the filter to extract the parameters of a small object in a complex scene. The sphere has a radius $a(\mathrm{~m})$. 
The actual initial state of the scene is: $\left(d_{x}=0.3 \mathrm{~m}, d_{y}=0.3 \mathrm{~m}, \theta_{w}=0 \mathrm{rad}, d=0.4 \mathrm{~m}, \theta=1.57\right.$ $\mathrm{rad}, a=0.0305 \mathrm{~m})$ and the initial estimated state of the scene is : ( $\hat{d}_{x}=d_{x}, \hat{d}_{y}=d_{y}, \hat{\theta}_{w}=\theta_{w}$, $\hat{d}=d-0.08 \mathrm{~m}, \hat{\theta}=\theta+0.03 \mathrm{rad}, \hat{a}=a-0.02 \mathrm{~m})$. Figure $13(\mathrm{~b})$ shows the real and estimated state of the corner with respect to time. Figure 13(a) shows the real and the estimated state of the sphere with respect to time. Figure 13(c) represents the actual and the estimated scene at different times. At $t=0 \mathrm{~s}$ the position of the real walls is known a priori (thus the initial estimate error for the wall state is null). For times between $0<t<8 \mathrm{~s}$, the sensor does not detect the sphere because it is too distant. At $t>8 \mathrm{~s}$ the sensor perceives the perturbation due to the sphere and the estimated parameters of the sphere converge toward their actual values. For times between $8<t<23 \mathrm{~s}$, the sensor is close to the sphere and for $t>20$ s the sphere is quite well localized. This test highlights that it is possible to distinguish (localize and reconstruct) a small object immersed in a complex scene, when the sensor is close to this object.
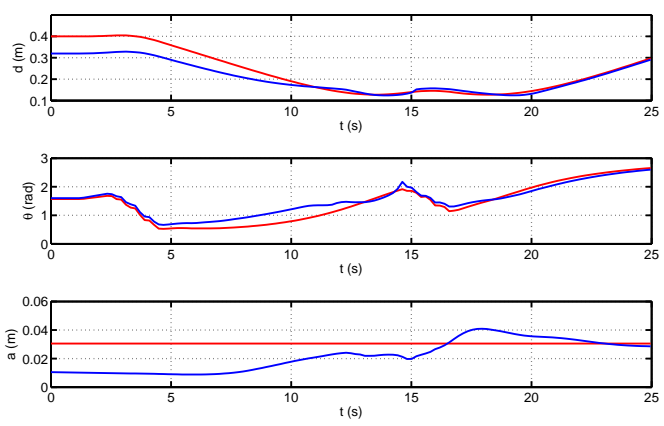
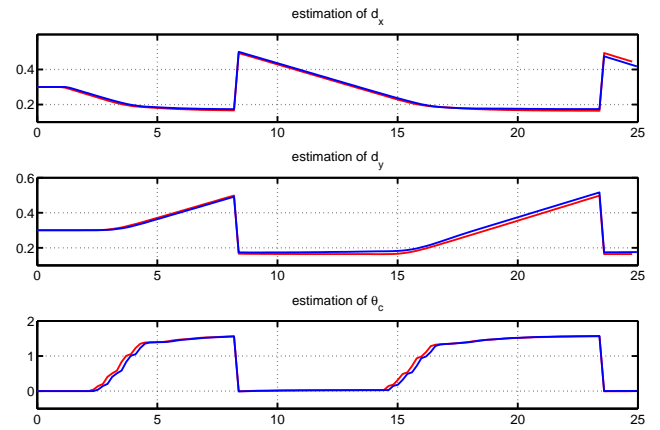

(a) Real (red) and estimated (blue) state of the sphere.

(b) Real (red) and estimated (blue) state of the corner.
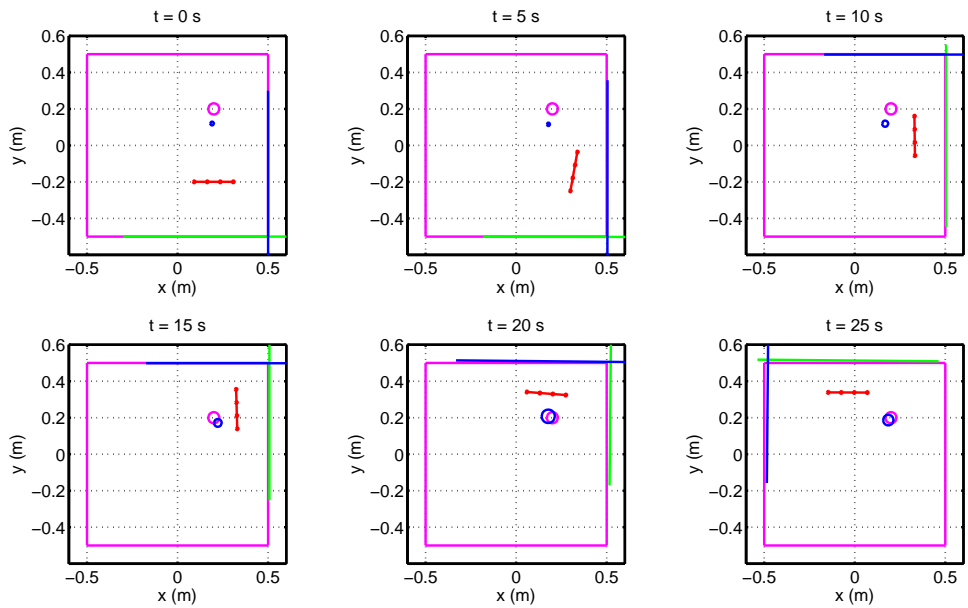

(c) Real (cyan) and the estimated (blue) scene.

Figure 13: Reconstruction of a sphere in a complex scene $\left(V_{\|}=0.04 \mathrm{~m} / \mathrm{s}\right)$. 


\section{Electro-navigation}

The problem of electro-navigation based on the knowledge of environment derived from the electric sense will now be addressed. The sensor is put into our test bench and the objective of the control law is to follow the walls of the tank at a given distance $d^{d}$. This distance must be less than the sensor range and large enough to avoid collision of the robot with the tank during the experiment, a value of $d^{d}=0.15 \mathrm{~m}$ is chosen. Due to the range of the sensor compared with the size of the tank, we designed a control law based on a local model composed of a corner only (see Figure $3(b))$. The state feedback in the control law uses the estimated state computed by the Kalman filter. In addition to the nominal task of following the walls, we also assessed the robustness of the solution.

\subsection{Control law}

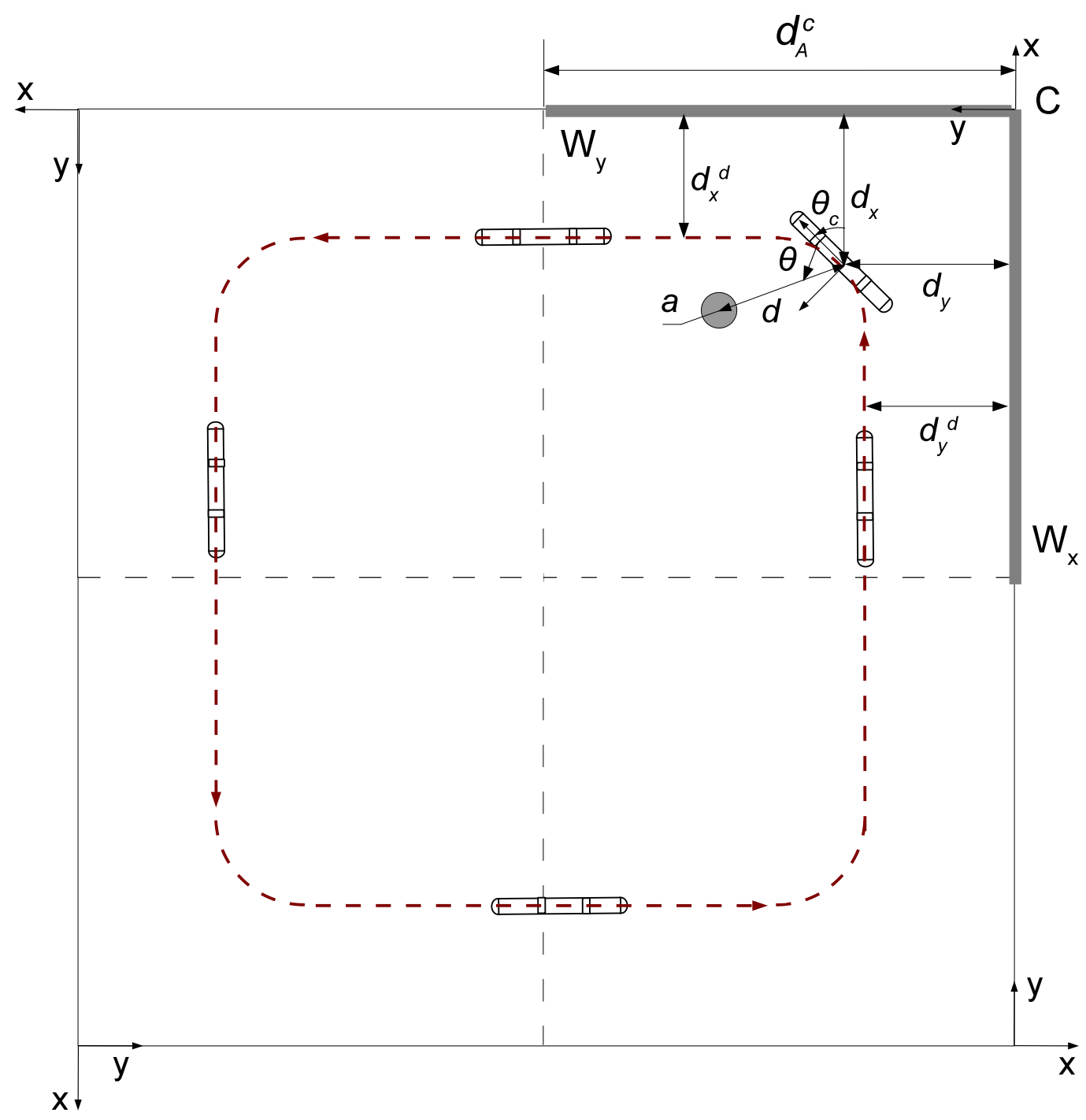

Figure 14: Parametrization of the sensor following the walls of the tank (the upper index "d" means "desired"). 
The motion of the robot with respect to the environment is defined by equation (6). The control inputs are $V_{\|}$and $\Omega_{\perp}$. The control law uses the observed value with respect to the corner frame. Turning around the tank is first considered. The control task is decomposed into two sub-tasks (see Figure 14). Firstly, the robot has to follow the axis $X\left(\theta_{c}^{d}=0\right)$ at the distance $d_{y}^{d}=0.15 \mathrm{~m}$. Secondly, when the robot reaches the corner $\left(d_{x}<d_{x}^{c}=0.2 \mathrm{~m}\right)$, the robot has to follow the axis $Y,\left(\theta_{c}^{d}=\pi / 2\right)$ at the distance $d_{x}^{d}=0.15 \mathrm{~m}$. While following the walls, the forward velocity of the robot is kept constant, while the angular velocity $\Omega_{\perp}$ is controlled to achieve the task. Thus, the objective of the first task consists in forcing the two following outputs to equal zero:

$$
\left\{\begin{array}{l}
y_{1}=\theta_{c}^{d}-\theta_{c} \\
y_{2}=d_{y}^{d}-d_{y} .
\end{array}\right.
$$

To achieve this objective a simple proportional control law is proposed:

$$
\Omega_{\perp}=k_{d}\left(d_{y}^{d}-d_{y}\right)+k_{\theta}\left(\theta_{c}^{d}-\theta_{c}\right) .
$$

Using (6), the evolution of this distance $d_{y}$ is:

$$
\dot{d}_{y}=V_{\|} \sin \left(\theta_{c}\right)
$$

Deriving this equation and using (14), the behavior in closed loop becomes:

$$
\ddot{d}_{y}=V_{\|}\left(k_{d}\left(d_{y}^{d}-d_{y}\right)+k_{\theta}\left(\theta_{c}^{d}-\theta_{c}\right)\right) \cos \left(\theta_{c}\right) .
$$

The linearization of (15) and (16) around the desired position $\left(\theta_{c}^{d}=0\right)$ gives:

$$
\left\{\begin{array}{l}
\dot{d}_{y}=V_{\|} \theta_{c}, \\
\ddot{d}_{y}=V_{\|} k_{d}\left(d_{y}^{d}-d_{y}\right)-V_{\|} k_{\theta}\left(\theta_{c}\right) .
\end{array}\right.
$$

By combining the first equation with the second, we find:

$$
\ddot{d}_{y}=-k_{\theta} \dot{d}_{y}-V_{\|} k_{d}\left(d_{y}^{d}-d_{y}\right)
$$

The gains $k_{\theta}$ and $k_{t}$ can be easily chosen to ensure the convergence of $d_{y}$ to $d_{y}^{d}$ for any fixed velocity $V_{\|}$. Using (14), it is clear that $\theta_{c}$ will also converge toward its desired value. In the experiment, the values chosen are $V_{\|}=0.04 \mathrm{~m} / \mathrm{s}, k_{\theta}=2, k_{d}=25$. To follow the second wall, a similar control law is used, but it is the distance $d_{x}$ that is controlled in this case and not $d_{y}$. Thus, the control law becomes:

$$
\Omega_{\perp}=k_{d}\left(d_{x}^{d}-d_{x}\right)+k_{\theta}\left(\theta_{c}^{d}-\theta_{c}\right),
$$

with $\theta_{c}^{d}=\pi / 2$. Note that when the robot reaches the corner, the desired values $\theta_{c}^{d}$ and $d_{y}^{d}$ are not instantaneously switched to their desired values $\theta_{c}^{d}=\pi / 2$ and $d_{y}^{d}=0.15 \mathrm{~m}$. This avoids the large variations of $\Omega_{\perp}$ which would occur in this case. In fact, $\theta_{c}^{d}(t)$ and $d_{y}^{d}(t)$ are parameterized with cubic polynomials allowing then to commute smoothly from one subtask to the other. This is achieved over 30 sampling periods each of $15 \mathrm{~ms}$.

Despite its apparent specificity, this control law has a certain generality. For instance, it also works when the walls are not straight but smoothly and moderately curved. Indeed, in this case, (14) can be used with $y$ defining now the instantaneous tangent to the wall, with respect to which the sensor is positioned at a distance $d_{y}$ and tilted of an angle $\theta_{c}$. Furthermore, in the case where 
the curvature of the followed wall becomes too high or even presents some discontinuities, the same control law can be used by introducing a $90^{\circ}$ corner. In this case the law will still work, as long as the variations of the corner angle do not exceed $\pm 20^{\circ}$, as we have seen on experiments with different orientations of a removable wall suddenly placed in the corners of our tank. Finally, going beyond this limit would need to come back to the modeling of the corner. Indeed, while in the case of obtuse angles the method of images still applies, in the case of acute angles, more sophisticated approaches based on the expansion of electric field on orthogonal functional basis would be required [Jak99].

\subsection{Change of observed model and of control law}

Allowing for the range of the sensor, the following strategy is proposed (see Figure 15) to achieve the task of following the walls. When the robot follows the $Y$-axis, it departs from the corner and the effect of the rear wall $W_{X}$ along the $X$-axis decreases. When the effect of $W_{X}$ becomes negligible (around $d_{y} \geqslant d_{A}^{c}=0.7 \mathrm{~m}$, see section 3.2), the local model used by the filter considers a new front wall. This is simply achieved by rotating the frame of the previous corner by $\pi / 2$. Then, the $X$ and $Y$ axes are permuted and the task "following a wall along $X$ " becomes "following a wall along $Y$ " with $d_{x}$ replacing $d_{y}$. Note that since the size of the tank is unknown, the value of $d_{x}$ is unknown too. The corresponding initial value of $d_{x}$ is fixed to the sensor range $d_{A}^{c}$ of section 3.2. The weighting matrix associated with $d_{x}$ is initialized with a large value since the estimation error is high in this case. The control law is thus decomposed into 2 tasks that are used alternatively as shown in Figure 15. Note that the commutation of control law is based on the observed values $d_{x}$ and $d_{y}$, and the local model is redefined after re-initialization of the observers when $d_{y}>d_{A}^{c}$. In

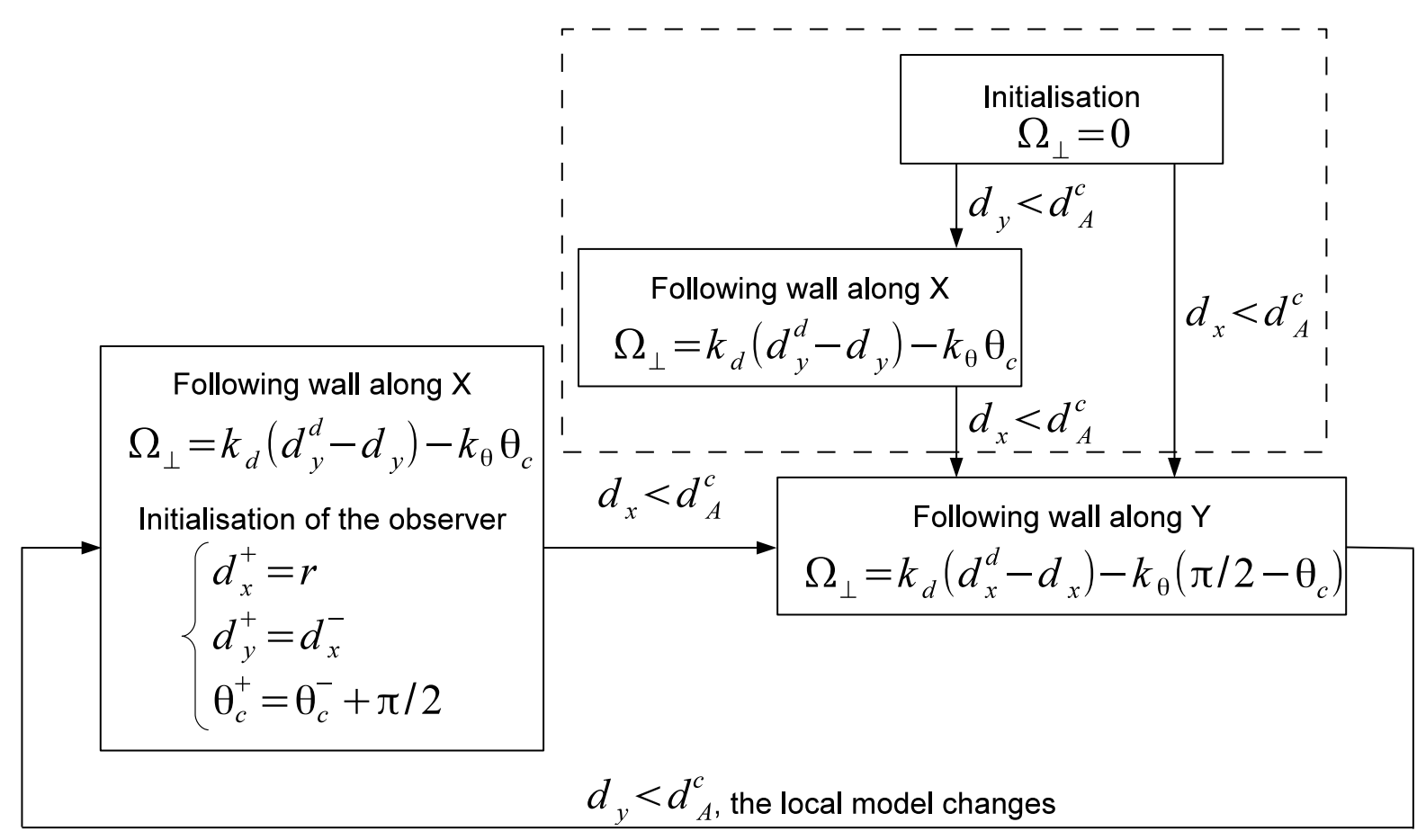

Figure 15: The complete control strategies including change of tasks and of local models. The turn around the tank is described by the loop in the lowest part of the figure. The part in the dotted box corresponds to the initialization of the motion of the robot in the tank. 
the general case, the robot is initially not on the desired trajectory. It first moves straight forward until it reaches a wall. Then, it follows this wall as shown in Figure 15. Finally, let us make the following further remarks:

- In Figure 15, the superscripts "-" and "+" are used to denote the observed state "just before" and "just after" the commutation of local models.

- In principle, in the control strategy, $d_{A}^{c}$ denotes the range at which the sensor detects a front wall (i.e. a corner). However, since the length of a tank side is less than twice the sensor range, in practice we artificially decrease the range to $d_{A}^{c}=0.5 \mathrm{~m}$.

More fundamentally, due to the limited range of our sensor, it is artificial and even dangerous to use a global model of the environment. Indeed, when no measurement is available to inform one part of such a global model, the integration of the motion model leads to a drift of the model of the environment. On the other hand, when using a local model of the environment, it is in general difficult to consider a convenient local model. In fact, if it is easy to remove an object from the local model when it becomes out of range for the sensor, it is much more difficult to add a new object entering the detection bubble of the sensor. In particular, we have to define what new object we have to add to the scene and when it has to be added. Up till now, we did not get answers to all of those questions in the general case. In our particular case, the task consists in following the tank boundaries on the right side of the sensor. To achieve this task, only corners were considered as local models for the sensor. The commutations between these local models were managed as follows. As soon as the wall in the back of the sensor becomes out of its range, it is removed from the model and replaced by a front wall (this corresponds to replace a first model of a corner by a second one rotated of $90^{\circ}$ with respect to the first). Till the front wall is not sensed by the robot its distance from the sensor will remain at a value higher than the range. This approach can be used to follow the boundary of any bounded space without any object.

\subsection{Following a wall.}

The 2-electrode sensor's trajectory follows the wall at a constant distance $\left(d^{d}=0.15 \mathrm{~m}\right)$. As shown in $\left[\mathrm{LCA}^{+} 10\right]$, a yawing motion increases the convergence of the Kalman filter. The yawing motion is introduced by adding a harmonic component to $\Omega_{\perp}$ comparable to that observed in the swimming gait of the fish. The amplitude of this yawing oscillation is $10^{\circ}$ and its frequency is $0.2 \mathrm{~Hz}$. Figure 16(a) shows the sensor trajectory with and without yawing and Figure 16(b) shows the real and estimated state. The sensor starts from a random point (point A), the feedback control law uses the relative position of the sensor with respect to the environment (corner) estimated by the Kalman filter. For as long as it has not detected the wall, the sensor goes straight forward (A). As soon as a wall is detected, the sensor starts to follow it (from B to C). When the distance between the sensor and the wall in front of it is equal to a desired distance, the sensor turns around the corner (from $\mathrm{C}$ to $\mathrm{D}$ ). The result shows that we can use the 2-electrode device to achieve a basic task such as following a wall. 


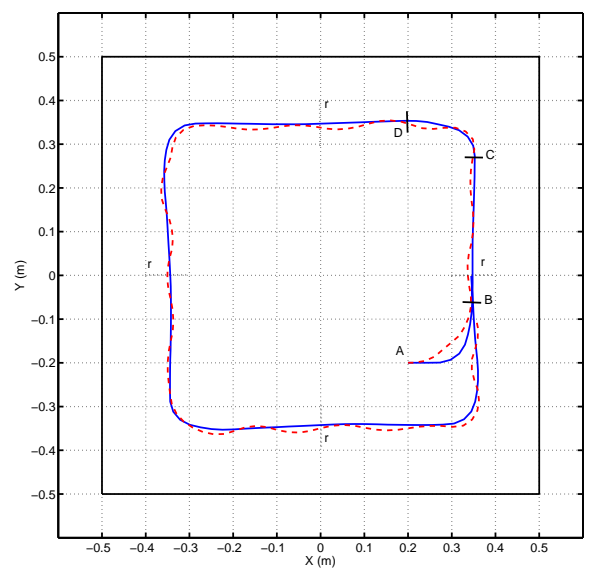

(a) Sensor's center trajectory. The blue line is the trajectory without yawing and the dotted red line is the trajectory with yawing.

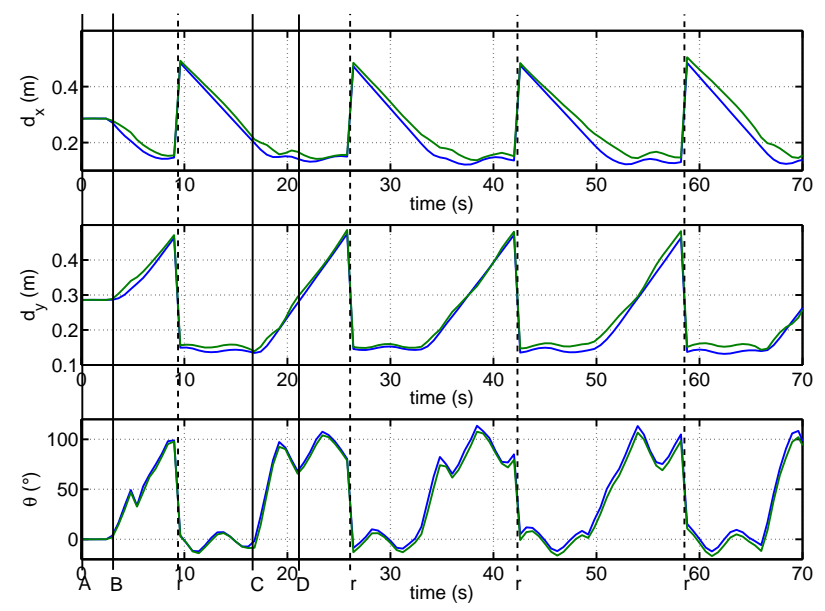

(b) Estimated and measured states with yawing.

Figure 16: Motion in straight line with and without yawing $\left(V_{\|}=0.04 \mathrm{~m} / \mathrm{s}\right.$ and $\left.d^{d}=15 \mathrm{~cm}\right)$.

\subsection{Robust test: removable wall}

In this test, we made 2 laps of the tank (Figure 17) and during the second lap (dotted line), we put a removable wall (wall marked with dotted line on Figure 17) at a distance of $0.1 \mathrm{~m}$ from the first one. Then, the tank $1 \times 1 \times 1 \mathrm{~m}$ becomes a parallelepiped of $0.9 \times 1 \times 1 \mathrm{~m}$. The controlled sensor has no additional information and has to detect and follow the new wall (from A to B). Figure 18 shows the sensor position $\mathbf{x}=\left[d_{x}, d_{y}, \theta_{c}\right]$ measured with the Cartesian robot (in blue) and that estimated $\hat{\mathbf{x}}=\left[\hat{d}_{x}, \hat{d}_{y}, \hat{\theta}_{c}\right]$ with electric sense (in green). So when the sensor follows the removable wall (from $\mathrm{A}$ to $\mathrm{B}$ ) during the second lap, we show a gap of $0.1 \mathrm{~m}$ on $d_{x}$ (or $d_{y}$ depending on the corner reference), which is the distance between the removable and real tank wall. The 2-electrode sensor detects the new wall and turns (Figure 17(a)). However, close to points A and B (Figure 18(a)), the estimation of the scene's parameters $\hat{d}_{x}$ and $\hat{d}_{y}$ are both simultaneously disrupted by the new wall. In the same conditions but using the 4-electrode sensor (Figure 18(b)), only one of the estimated parameters $\hat{d}_{x}$ or $\hat{d}_{y}$ is disrupted. This result shows that for the 2 and 4-electrode sensors, the closed feedback control loop is robust to the variations of the wall's position. 


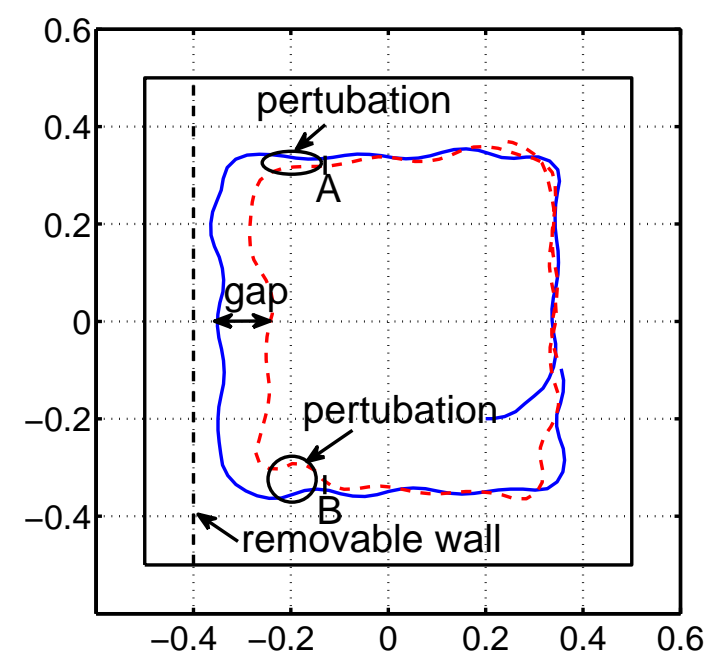

(a) 2-electrode probe.

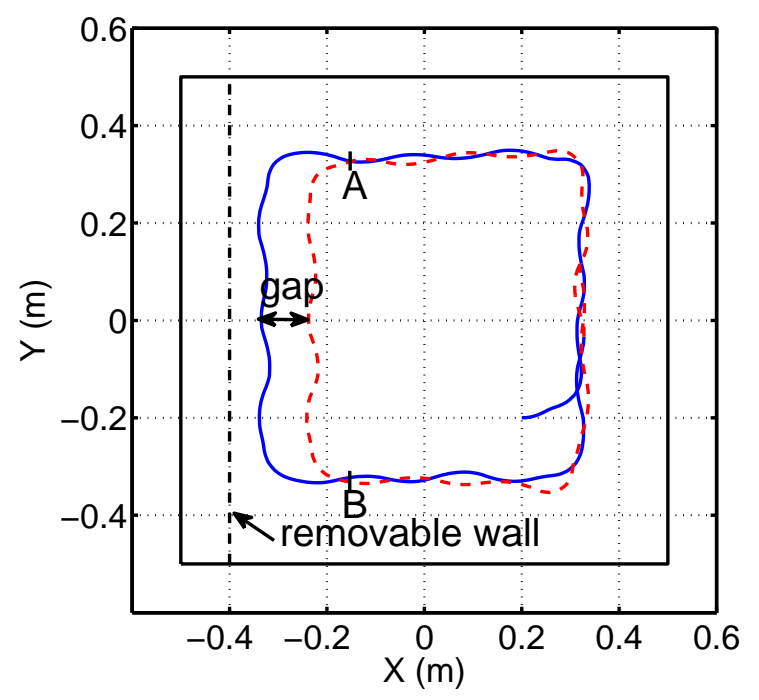

(b) 4-electrode probe.

Figure 17: Turning a corner with removable wall $\left(V_{\|}=0.04 \mathrm{~m} / \mathrm{s}\right.$ and $\left.d^{d}=15 \mathrm{~cm}\right)$. The blue line is the trajectory without the wall and the dot red line is the trajectory with the wall.

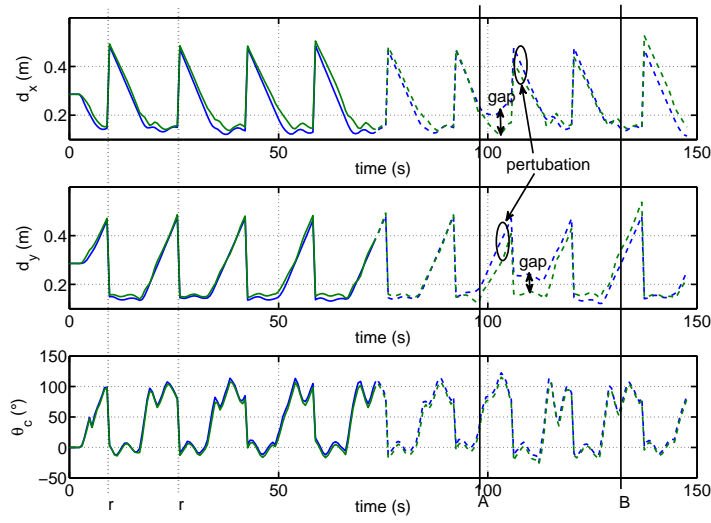

(a) 2-electrode probe.
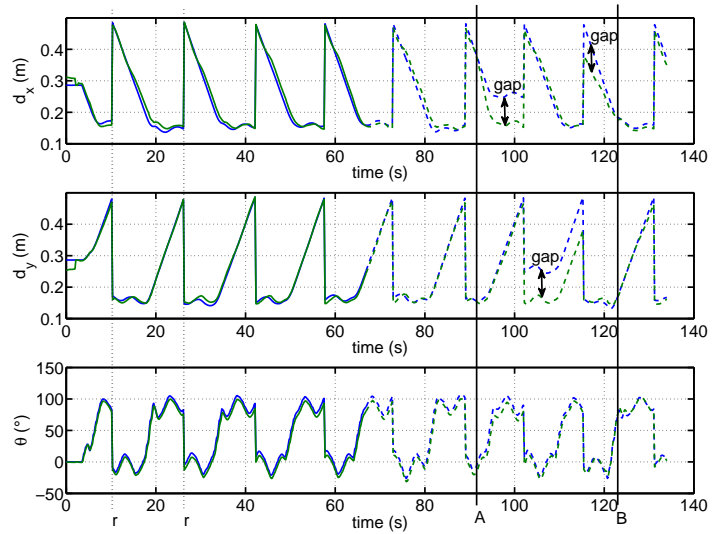

(b) 4-electrode probe.

Figure 18: Estimated and measured states with an additional wall. The line is the error without the wall and the dot line is the error with the wall.

\subsection{Robust test: wall with lump}

The aim of this test is to assess the robustness of the law to an un-modeled perturbative object. For that purpose, we placed a small insulating sphere (with a radius $=0.0325 \mathrm{~m}$ ) in the tank close to a wall (on the previous sensor's trajectory). As in the previous case, the local model used by the filter is that of a corner and the additional sphere is not considered in the model. Figure 19(a) shows the trajectory of the 2-electrode sensor with the sphere in its path. At the beginning the sensor is not perturbed by the sphere (which can be considered as a lump on the wall). The sensor 
moves forward until it reaches the desired distance from the wall, then it follows the wall as in the previous tests. When the sensor follows the wall on which there is a lump, the measured current progressively decreases because of the insulating sphere. The estimated parameters $\hat{d}_{x}, \hat{d}_{y}$ decrease and the sensor moves away. The control law ensures a constant distance between the sensor and the wall. When the sphere is between the two electrodes of the 2-electrode sensor, it is in its blind spot, and so moves closer to the wall. Suddenly the sphere reappears in the sensor's range, decreasing the current. As a result, the sensor goes quickly away and collides with the sphere. Figure 19(b) displays the result of the same test but with the 4-electrode sensor. In this case, the sensor avoids the lump, which has never disappeared from its range. Thus, this test shows that increasing the measurement surface on the sensor increases the robustness of the navigation.

While the global behaviors of the navigation are satisfactory, due to the closed loop structure it is in general difficult to separate the effect of the measurements from that of the control law. However, this experiment shows how the measurements can have a strong influence on the global performances of the navigation. Let us also note that this test is only a robustness test since the control law is not designed to cope with the presence of a perturbative object. Consequently, a correct behavior cannot be obtained for any position of the object. In the general case, the proposed control law (based on a model of the environment) could be mixed with a reflex controller allowing to avoid obstacles through fast reactive laws which directly feedback the electric measurements [LBCS12].

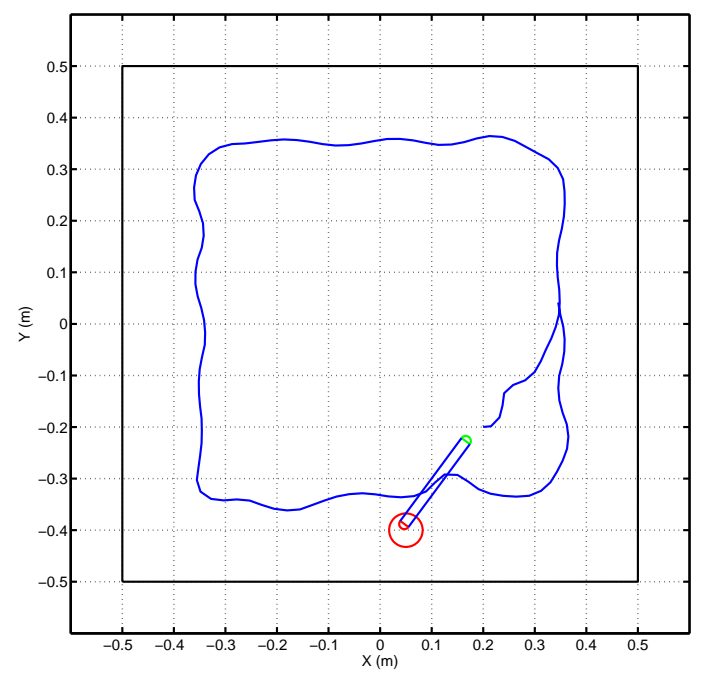

(a) Additional sphere on the sensor's trajectory with the 2-electrode probe

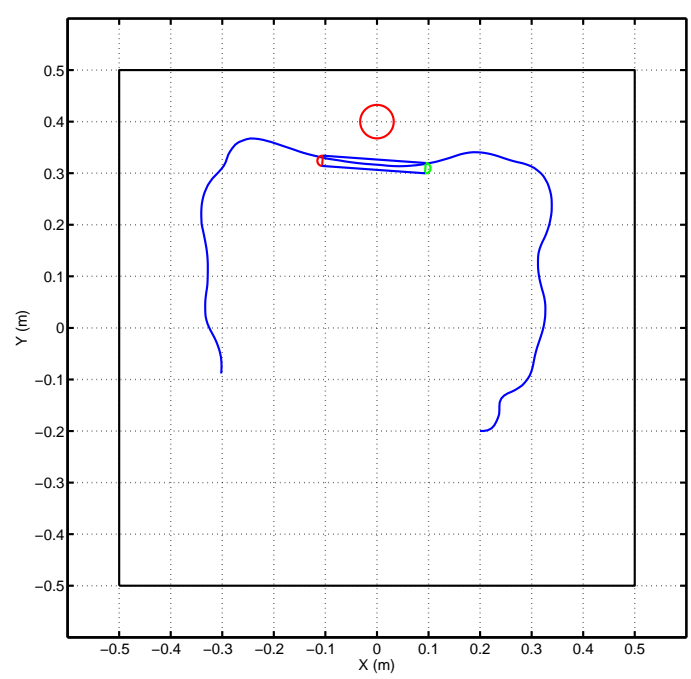

(b) Additional sphere on the sensor's trajectory with the 4-electrode probe

Figure 19: Robustness tests $\left(V_{\|}=0.04 \mathrm{~m} / \mathrm{s}\right.$ and $\left.d^{d}=15 \mathrm{~cm}\right)$. An insulating sphere is suddenly placed on the path of the sensor. 


\section{Conclusion and perspectives}

Using a sensor inspired by electric fish, we have shown experimentally that a 2-electrode probe is capable of navigating in a tank with flat walls, but when an obstacle (lump) appears on the sensor's trajectory, the sensor cannot avoid it. This drawback is essentially due to the lateral range of the sensor which is limited by the configuration of the electrodes (number and location) and the presence of blind spots. However, upgrading to a 4-electrodes sensor leads to the disappearance of these blind spots and enables the sensor to avoid an un-modeled object located on its trajectory.

We have shown that small objects can be reconstructed and localized as equivalent spheres in both simple and complex scenes. These results are encouraging, but leave much future work to be pursued. For instance, the differences in measured current between a sphere and a cube are too small to allow the Kalman filter to distinguish between them. Another way to improve the results presented here concerns the used of the richness of the sensor measurements. With the current sensor design the reconstruction of a sphere (or a cube) suffers from intrinsic ambiguity due to the symmetry of the sensor. For instance, two identical objects located symmetrically on both side of the sensor will generate the same measurements. This scenario is illustrated on Figure 20(b), where the estimated state of the scene is initialized to a symmetric position so leading to a reconstructed sphere symmetric to the real sphere with respect to the sensor axis. In order to disambiguate this kind of situation, we developed a sensor in which the electrodes are separated into two lateral (left and right) measurement sub-electrodes [BBG08, BGJ $\left.{ }^{+} 12\right]$. Among its advantages, this "binocular" sensor allows for the identification of the electric nature (insulating or conducting) of the material used for the objects, thus releasing us from the need for a priori knowledge of $\gamma$ and $\gamma_{s}$. The implementation of these models in the unscented Kalman filters is now in progress.

This article has presented our current approach to the reconstruction and navigation problems. As a further step we will have to include this filter in a global control feedback loop which will allow a real underwater robot to navigate in a complex scene. Towards this goal we have recently proposed another solution to the navigation problem. Based on sensor based control laws, this alternative reactive approach requires no model of the scene and considers the navigation problem as a low level reflex control problem [BLCS12].
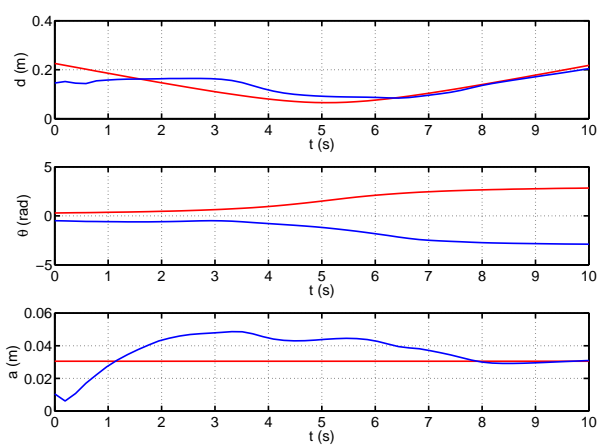

(a) Real (red) and estimated (blue) state of the sphere.
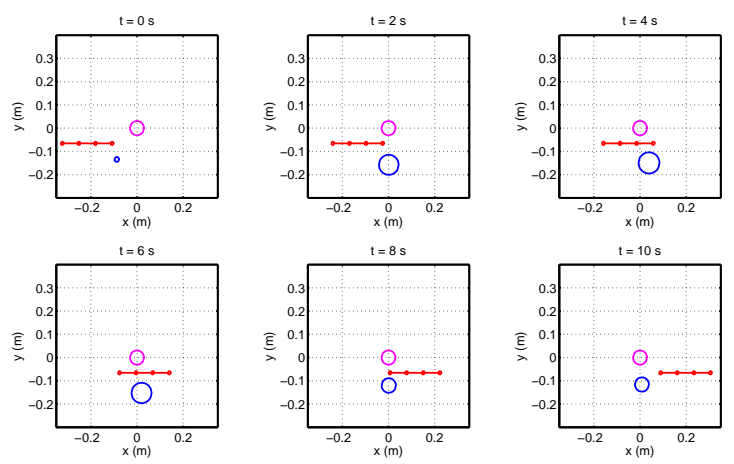

(b) Real (cyan) and the estimated (blue) scene.

Figure 20: Reconstruction of a symmetric sphere in a simple scene. 
Finally, beyond these first results, many things remain to be done before equipping an operational underwater vehicle with electric sense. In this perspective, the Angels project [Ang09] has developed small underwater robots (modules) capable of navigating in swarm (see Figure 21). Recently, we have implemented the electric sense on these robots. As expected the range of electric sense is about the length of the modules. Remarkably, first experiments in salty water show that the same range can be obtained in sea water with slight adaptation of the electronic measurements [SJB $\left.{ }^{+} 11\right]$. Currently, we are implementing reactive controllers [BLCS12, LBCS12] for obstacle avoidance on our modules. In the future, we will apply the control laws and scene reconstruction techniques described above to perform autonomous mapping of an unknown environment.
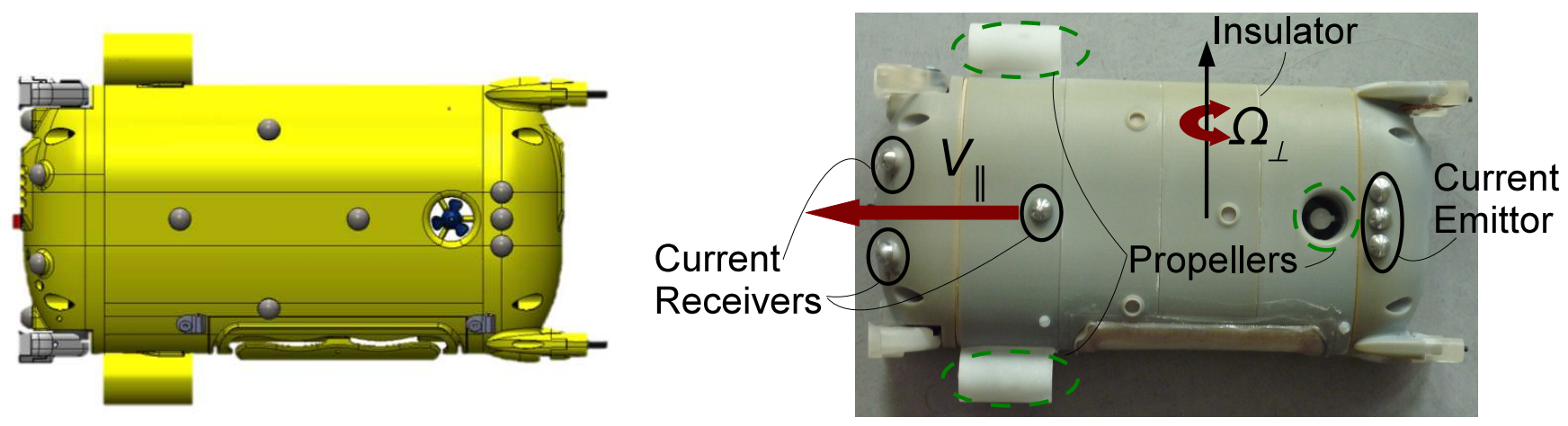

Figure 21: The ANGELS module (left CAD, right real robot), designed by the CRIM Lab of Scuola Superiore Sant'Anna. The small hemispherical electrodes should be noted. The module is axially propelled $\left(V_{\|}\right)$by two top and down propellers. One lateral propeller crosses laterally the body and ensures the steering $\left(\Omega_{\perp}\right)$.

\section{Acknowledgments}

The ANGELS project is funded by the European Commission, Information Society and Media, Future and Emerging Technologies (FET) contract number: 231845. The authors gratefully acknowledge Stéphane Bouvier ${ }^{\ddagger}$ for his contribution to the development of the electronics of the electrolocation and test bench, and the assistance of Douglas Carnal in the preparation of this article for publication.

\section{References}

[Ang09] The angels' project, http://www.theangelsproject.eu, 2009.

[BBG08] G. Baffet, F. Boyer, and P.B. Gossiaux. Biomimetic localization using the electrolocation sense of the electric fish. In Robotics and Biomimetics ROBIO IEEE, 2008.

$\left[\mathrm{BGJ}^{+} 12\right] \quad$ F. Boyer, P.B. Gossiaux, B. Jawad, V. Lebastard, and M.Porez. Model for a sensor inspired by electric fish. IEEE transactions on robotics, 28(2):492-505, 2012.

[BLCS12] F. Boyer, V. Lebastard, C. Chevallereau, and N. Servagent. Underwater reflex navigation in confined environment based on electric sense. IEEE transactions on robotics, submitted 2012. 
[CBGB98] A. Caputi, R. Budelli, K. Grant, and C. Bell. The electric image in weakly electric fish: Physical images of resistive objects in gnathonemus petersii. Journal of Experimental Biology, 201:pp. 2115-2128, 1998.

[CHKN05] L. Chen, J. House, R Krahe, and M. Nelson. Modeling signal and background components of electrosensory scenes. J Comp. Physiol A, 191:pp. 331-345, 2005.

$\left[\mathrm{EBM}^{+} 08\right]$ Jacob Engelmann, Joäo Bacelo, Michael Metzen, Roland Pusch, Beatrice Bouton, Adriana Migliaro, Angel Caputi, Ruben Budelli, Kirsty Grant, and Gerhard von der Emde. Electric imaging through active electrolocation: implication for the analysis of complex scenes. Biol. Cybern., 98(6):519-539, May 2008.

[EvdE11] Jacob Engelmann and Gerhard von der Emde. Active electrolocation. Encyclopedia of Fish Physiology: From Genome to Environment., pages 375 - 386., 2011.

[Jak99] John David Jakson. Classical Electrodynamics 3rd edition. John Wiley and Sons, 1999.

$\left[\mathrm{JGB}^{+} 10\right] \quad$ B. Jawad, P.B. Gossiaux, F. Boyer, V. Lebastard, F.Gomez, N.Servagent, S.Bouvier, A.Girin, and M.Porez. Sensor model for the navigation of underwater vehicles by the electric sense. In Robotics and Biomimetics ROBIO IEEE, 2010.

[Kal60] R.E. Kalman. A new approach to linear filtering and prediction problems. Transactions of the ASME - Journal of Basic Engineering, 82:pp. 35-45, 1960.

[LBCS12] Vincent Lebastard, Frédéric Boyer, Christine Chevallereau, and Noël Servagent. Underwater electro-navigation in the dark. In IEEE International Conference on Robotics and Automation, ICRA 2012, pages 1155-1160, St. Paul, Minnesota, USA, 14-18 May 2012. IEEE.

$\left[\mathrm{LCA}^{+} 10\right] \quad$ V. Lebastard, C. Chevallereau, A. Amrouche, B. Jawad, A. Girin, F. Boyer, and P.B. Gossiaux. Underwater robot navigation around a sphere using electrolocation sense and kalman filter. In IROS 2010 IEEE, 2010.

[LM58] H.W. Lissmann and K.E. Machin. The mechanism of object location in gymnarchus niloticus and similar fish. The Journal of Experimental Biology, (35):451-486, 1958.

[NdS90] H. Nijmeijer and A.J. Van der Schaft. Nonlinear Dynamical Control Systems. Springer-Verlag, 1990.

[PLIB11] M. Porez, V. Lebastard, A. J. Ijsperet, and F. Boyer. Multi-physic model of an electro fish-like robot : numerical aspect and application to the obstacle avoidance. In IROS 2011, 2011.

[Ras96] B. Rasnow. The effects of simple objects on the electric field of apteronotus. Journal of Comparative Physiology A, 3(178):397-411, 1996.

[SJ97] J. Julier Simon and K. Uhlmann Jeffrey. A new extension of the kalman filter to nonlinear systems. In Int. Symp. Aerospace/Defense Sensing, Simul. and Controls, pages 182-193, 1997. 
[SJB $\left.{ }^{+} 11\right] \quad$ N. Servagent, B. Jawad, S. Bouvier, F. Boyer, A. Girin, F. Gomez, V. Lebastard, and P.-B. Gossiaux. First results on a sensor bio-inspired by electric fish. IEEE SENSORS JOURNAL, submitted (http://hal.archives-ouvertes.fr/hal-00695453), 2011.

[SLM07] J.R. Solberg, K.M. Lynch, and M.A. MacIver. Robotic electrolocation: Active underwater target localization. International Conference on Robotics and Automation, 2007.

[SLM08] J. Solberg, K. Lynch, and M. MacIver. Active electrolocation for underwater target localization. The International Journal of Robotics Research, 27:529-548, 2008.

[SYOPA04] A. Sihvola, P. Ylä-Oijala, S. Järven Pää, and J. Avelin. Polarizabilities of platonic solids. IEEE transactions on antennas and propagation, 52(9), 2004.

[vdE99] G. von der Emde. Active electrolocation of objects in weakly electric fish. The Journal of Experimental Biology, 202:1205-1215, 1999.

[vdE06] Gerhard von der Emde. Non-visual environmental imaging and object detection through active electrolocation in weakly electric fish. Journal of Comparative Physiology A, 192:601-612, 2006.

[vdESG $\left.{ }^{+} 98\right]$ G. von der Emde, S. Schwarz, L. Gomez, R. Budelli, and K. Grant. Electric fish measure distance in the dark. Letters to Nature, Nature, 395:890-894, 1998. 